\title{
A SUPPLY AND DEMAND FRAMEWORK FOR TWO-SIDED MATCHING MARKETS
}

\author{
EDUARDO M. AZEVEDO AND JACOB D. LESHNO
}

\begin{abstract}
We propose a new model of two-sided matching markets, which allows for complex heterogeneous preferences, but is more tractable than the standard model, yielding rich comparative statics and new results on large matching markets.

We simplify the standard Gale and Shapley (1962) model in two ways. First, following Aumann (1964) we consider a setting where a finite number of agents on one side (colleges or firms) are matched to a continuum mass of agents on the other side (students or workers). Second, we show that, in both the discrete and continuum model, stable matchings have a very simple structure, with colleges accepting students ranked above a threshold, and students demanding their favorite college that will accept them. Moreover, stable matchings may be found by solving for thresholds that balance supply and demand for colleges. We give general conditions under which the continuum model admits a unique stable matching, in contrast to the standard discrete model. This stable matching varies continuously with the parameters of the model, and comparative statics may be derived as in competitive equilibrium theory, through the market clearing equations. Moreover, given a sequence of large discrete economies converging to a limit economy, the set of stable matchings of the discrete economies converges to the stable matching of the limit economy.

We bound the rate of convergence of the set of stable matchings of large discrete economies to the continuum approximation, and show that comparative statics regarding the unique stable matching of the continuum model extend to strong set ordering of the sets of stable matchings of approximating discrete economies. We model the transferrable utility case, as in Becker (1973). We characterize the limit of school choice mechanisms used in practice, generalizing previous results of Che and Kojima (2010). Finally, we illustrate the model's applicability by quantifying how competition induced by school choice gives schools incentives to invest in quality. Specifically, we show that schools have muted, and possibly even negative incentives to invest in quality dimensions that benefit lower ranked students.
\end{abstract}

Date: November 26, 2011.

Azevedo: Corresponding author, Harvard University azevedo@fas.harvard.edu. Leshno: Harvard University and Harvard Business School leshno@hbs.edu. An earlier draft was circulated as "College Admissions with a Continuum of Students". For comments on the earlier and present version we would like to thank Charles Angelucci, Itai Ashlagi, Dan Bjorkegren, Gabriel Carroll, Carlos da Costa, Drew Fudenberg, Oliver Hart, Thomas Kahle, Fuhito Kojima, Scott Kominers, Jose Montiel, Humberto Moreira, Alex Nichifor, Muriel Niederle, Ilya Segal, Glen Weyl, Yosuke Yasuda and seminar participants at Harvard and the Booth School of Business. We would like to thank specially Susan Athey, Eric Budish, and Alvin Roth for many helpful suggestions. Any remaining mistakes are our own. 


\section{INTRODUCTION}

1.1. Overview. In two-sided matching markets buyers and sellers have preferences over who they interact with on the other side of the market. For example, consulting firms competing for college graduates care about which workers they hire. Such a market clears not only through wages, as a college graduate cannot simply demand the firm he prefers - he must also be chosen by the firm. These are key features of many important markets, and matching markets have been extensively studied in Economics. Much of the literature is based on one of two classic frameworks, each with distinct advantages and limitations. ${ }^{1}$

One strand of the literature follows Becker's (1973; 1974) marriage model. These models often assume simple preferences, with men and women being ranked from best to worst. Moreover, utility is transferable, so that a couple may freely divide the gains from marriage. These stark assumptions lead to simple models with rich comparative statics that have been applied to diverse problems such as explaining sex differences in educational attainment, changes in CEO wages, and the relationship between the distribution of talent and international trade. ${ }^{2}$

Another line of research follows Gale and Shapley's (1962) college admissions model. These models allow for complex heterogeneous preferences, and (possibly) for limitations on how parties may divide the surplus of a relationship. Due to its generality, this model is a cornerstone of market design, and has been applied to the study and design of market clearinghouses (e.g. the National Resident Matching Program, that matches 30,000 doctors and hospitals per year; the Boston and New York City public school matches, which match over 100,000 students per year), ${ }^{3}$ the use of signaling in labor markets, ${ }^{4}$ the relationship between matching and auctions, ${ }^{5}$ and supply chain networks. ${ }^{6}$ This framework has had less success in empirical applications, where the multiplicity of solutions is an issue, ${ }^{7}$ and in obtaining comparative statics results. In contrast to the

\footnotetext{
${ }^{1}$ In this paper we focus on frictionless matching markets, where the appropriate equilibrium concept is the notion of stability defined below. Another important literature pioneered by Mortensen and Pissarides (1994), considers matching markets with frictions. We do not pursue this line of inquiry.

${ }^{2}$ See respectively Chiappori et al. (2009); Gabaix and Landier (2008) and Tervio (2008); Grossman (2004), and the related paper by Grossman and Maggi (2000).

${ }^{3}$ The redesign of the National Resident Matching Program is described in Roth and Peranson (1999). School choice was introduced as a mechanism design problem in the seminal paper of Abdulkadiroglu and Sönmez (2003), and the redesign of the Boston and New York City matches is described in Abdulkadiroglu et al. (2005a,b).

${ }^{4}$ Coles et al. 2010, 2011; Lee et al. 2011.

${ }^{5}$ Hatfield and Milgrom 2005.

${ }^{6}$ Ostrovsky 2008.

${ }^{7}$ See Sorensen (2007) for a discussion of the issue of multiplicity of stable matchings for the estimation of matching models, and a set of assumptions under which it can be circumvented. The Gale and Shapley model is often used for the simulation of outcomes once preferences have been estimated, as in Lee et al. (2011) and Hitsch et al. (2010).
} 
former strand of the literature, comparative statics results are often difficult and of a limited nature in this framework. ${ }^{8}$

This paper proposes a new model of matching markets based on Aumann's (1964) insight that markets with a continuum of traders may be considerably simpler than those with a finite number of traders. Like the Gale and Shapley (1962) framework the proposed model allows for rich heterogeneous preferences and (possible) restrictions on transfers. However, like the Becker (1973) model, it also permits straightforward derivation of comparative statics. Finally, the new model implies new results on matching markets with a large but finite number of agents, and we apply it to examine the effects of competition among public schools on school quality.

The basic features of our model follow the standard Gale and Shapley college admissions model. Agents on one side (colleges or firms) are to be matched to many agents on the other side (students or workers). Throughout most of the paper we consider the extreme case where there are no transfers between agents. As in the standard model, the solution concept is stability. A matching between students and colleges is stable if no pair of a student and a college would like to break away from a match partner and match to each other.

We make two key simplifications to the standard Gale and Shapley model. First, we consider a model where each of a finite number of colleges is matched, not to a discrete number of students, but to a continuum mass of students. Therefore, our model approximates a situation where each agent on one side is matched to a large number of agents on the other side. This is the case in a number of important matching markets, such as college admissions, school choice clearinghouses, diverse labor markets (e.g. the market for associates at major US law firms), ${ }^{9}$ and debt and equity underwriting. ${ }^{10}$ The assumption of a continuum of students and finite number of colleges is similar to Aumann's (1964) use of a continuum of consumers trading finitely many types of goods.

The second key simplification is applying the logic of supply and demand to matching markets. We give a new characterization of stable matchings that allows us to use standard techniques from competitive equilibrium models in a matching setting. We

\footnotetext{
${ }^{8}$ For example, Crawford's (1991) main results are comparative statics theorems. He shows, in a generalization of the Gale and Shapley model, that adding a firm to the economy makes the worker-optimal and the firm-optimal stable matchings better for all workers. Hatfield and Milgrom's Theorem 6 shows that, in their model, a similar result is true when a worker leaves the market. Both these results, although unsurprising, are nontrivial, and the proofs depend on the Gale Shapley algorithm and its generalizations. In contrast, in our model comparative statics are obtained simply by differentiating the market clearing equations. This reveals not only the direction but also the magnitude of changes, and gives a systematic technique to derive comparative statics, which works in cases that may be less straightforward than the addition of a worker or firm.

${ }^{9}$ See Ginsburg and Wolf (2003) and Oyer and Schaefer (2010) for a description of institutional features of this market.

${ }^{10}$ This market is discussed in Asker and Ljungqvist (2010).
} 
show that, in both the continuum and the discrete models, stable matchings have a very simple structure, given by admission thresholds $P_{c}$ at each college $c$. We term such a threshold a cutoff, and colleges are said to accept students who are ranked above the cutoff. Given a vector of cutoffs P, a student's demanded college is defined as her favorite college that would accept her. We show that for every stable matching there exists a vector of cutoffs such that the demand function determines the match of each student. Moreover, at any vector of cutoffs $\mathrm{P}$ that clears supply and demand for colleges, the demand function yields a stable matching. Therefore, finding stable matchings in the Gale and Shapley model is equivalent to finding a solution $\mathrm{P}$ to a set of market clearing equations ${ }^{11}$

$$
D(P)=S .
$$

Mathematically, this is the same as solving a general equilibrium model, with cutoffs $\mathrm{P}$ in the stead of prices.

The continuum assumption considerably simplifies the analysis. First, in the continuum model, the correspondence between stable matchings and cutoffs that clear the market is one-to-one. Second, we show that with great generality the continuum model has a unique stable matching, and that this stable matching varies continuously with the underlying fundamentals. This result is in the spirit of Aumann's finding that the core allocation is unique in an economy with a continuum of traders. The results imply that comparative statics may be derived from the market clearing equations, using standard techniques. This is in contrast with the standard discrete model, that possibly has multiple stable matchings, which are typically found using the Gale and Shapley algorithm, rendering techniques such as the implicit function theorem not directly applicable.

To justify using the simpler continuum model as an approximation of real markets, we give a set of convergence results, which are our main theoretical contribution. A sequence of increasingly large discrete economies is said to converge to a continuum economy if the empirical distribution of student types converges to the distribution in the continuum economy, and the number of seats per student in each college converges. Whenever the continuum economy has a unique stable matching, all of the stable matchings of the discrete economies converge to this unique stable matching of the continuum economy. Moreover, we show that all stable matchings of the large discrete economies become very similar (in a sense we make precise). Therefore, even in a large discrete economy, the concept of stability clears the market in a way that is essentially unique.

Our paper is the first to characterize the asymptotics of the set of stable matchings in a class of large matching markets. As an application, we characterize the asymptotics

\footnotetext{
${ }^{11}$ As we discuss formally below, the formula $D(P)=S$ only holds when there is excess demand for all colleges. In general the system of equations to be solved is for every college $c: D_{c}(P) \leq S_{c}$, with equality if $P_{c}>0$.
} 
of school choice mechanisms used in practice to match students to schools, such as deferred acceptance with single tie-breaking. Previously, Che and Kojima (2010) had characterized the limit of the random serial dictatorship mechanism, and shown that it was identical to the probabilistic serial mechanism. This corresponds to the particular case of deferred acceptance with single tie-breaking where students do not have priorities to schools. Therefore, by allowing for more general preferences, our result covers the case actually used in practice where schools do give priorities to some students. Moreover, it gives a unified formula that describes the asymptotics of the important mechanisms of deferred acceptance with single tie-breaking, random serial dictatorship, probabilistic serial, and others that are covered by our model.

We illustrate the simple derivation of comparative statics within our framework by example. We consider how competition among schools induced by flexible school choice, as practiced in cities such as New York, gives incentives for schools to invest in quality. This problem has been previously studied in the discrete Gale and Shapley framework by Hatfield et al. (2011a). Using the continuum model, we give an expression for the effect for an individual school of investing in quality on the quality of its entering class. We decompose this effect in a direct effect, the gain from becoming more attractive keeping the admission thresholds of other schools fixed, and a market power effect, which is the loss from other schools becoming less selective. Using the decomposition, we show that schools have muted, or possibly even negative incentives to perform quality improvements that target lower ranked students. Therefore, while school choice might give schools incentives to improve, our result raises the concern that such improvements will disproportionately benefit top students.

Besides this simple example, two papers apply our model to derive Nash equilibria of games in matching markets where firms may be strategic. Crucially for these applications, the comparative statics in the continuum model deliver magnitudes of how a firm's actions impact market equilibrium. Azevedo (2011) considers what the distortions from market power are in imperfectly competitive matching markets, where firms may choose how much to invest in capacity. Veiga and Weyl (2011) analyze distortions in how platforms choose not only capacities, but different characteristics, such as how much colleges specialize.

For applications of the continuum model to real-life markets, it is important to determine how good an approximation it affords. We give bounds on how close the set of stable matchings of a large economy are to the stable matching in the continuum model. We consider the case where student types in a discrete economy are independently identically distributed according to a given measure. We show that the probability that cutoffs that clear the market deviate by more than a given constant from their limit value decreases exponentially with the size of the economy. Moreover, we bound the 
probability that the number of students that receive different matches than what is expected in the limit is greater than a constant, also by an exponentially decreasing quantity.

Likewise, it is important to guarantee that comparative statics results derived in the continuum model can be applied to discrete economies. We give a result showing that comparative statics in the continuum model extend to large discrete economies. Using cutoffs, we define orderings over stable matchings. We show that if the unique stable matchings of two continuum economies are ordered in a particular way, then the sets of stable matchings of two approximating large discrete economies are strongly set ordered in the same direction. Therefore, our model can be used to derive comparative statics in discrete economies. While directly proving such results often involves complex arguments, ${ }^{8}$ our model allows an analyst to simply apply the implicit function theorem to the continuum model, and the results are guaranteed to follow for large discrete economies.

Finally, in several markets wages or prices are personalized. We extend the model to a setting where parties (which in this case we term workers and firms) are free to negotiate contract terms, possibly including wages, in the spirit of Hatfield and Milgrom (2005) and Kelso and Crawford (1982). The set of possible contracts may have restrictions, so that the model encompasses the case of no transfers, as in the Gale and Shapley (1962) model, and the case of transferable utility, as in most of Becker's (1973) analysis. We define stability in terms of firm preferences over individual contracts, which is justified when preferences are responsive. We find that the simple characterization of stable matchings in terms of a small set of cutoffs still holds. Moreover, under fairly general conditions analogous to the case without transfers, the model yields a unique allocation of firms to workers in every stable matching. However, the division of surplus between firms and workers is no longer uniquely determined.

The paper is organized as follows. Section 1.2 clarifies the connections of our paper with previous work. Section 2 introduces our model (2.1), gives the new characterization of stable matchings in terms of supply and demand (2.2), and illustrates our main results through a simple example (2.3). Section 2 and the application in Section 5.1 are self-contained and sufficient for readers interested in applying our model.

Section 3 reviews the discrete Gale and Shapley model, and defines the notions of convergence we use. Section 4 proves our main theoretical results, giving conditions for the continuum economy to have a unique stable matching, and for convergence of the discrete model to our model. Section 5 discusses applications and extensions. Section 5.1 applies the model to understand how school competition gives schools incentives to improve quality, and what types of students are targeted by these improvements. Section 5.2 discusses bounds on our convergence results, 5.3 shows that comparative 
statics in the continuum model imply comparative statics in the discrete model, 5.4 derives asymptotic characterizations of assignment and school choice mechanisms, 5.5 considers economies with multiple stable matchings, and 5.6 extends the model to matching with contracts. Omitted proofs are in the Appendix.

1.2. Related Literature. Our paper is related to several active lines of research. First, our contribution is directly motivated by the literature on the design of large matching clearinghouses, such as the school matches in Boston and New York City (Abdulkadiroglu et al. 2005a,b). Such markets are both organized around clearinghouses, so that the resulting allocations are indeed stable, and have each school being matched to many students. One direct application of our model is as a framework to understand the properties of different mechanisms. In Section 5.4 we use our model to derive an asymptotic characterization of school choice mechanisms used in practice. In this vein, Azevedo and Leshno (2010) apply our model to perform an equilibrium analysis of a non-strategyproof mechanism suggested by Erdil and Ergin (2008).

In the mechanism design literature, there is a long tradition of studying the properties of large markets (Hurwicz 1972; Roberts and Postlewaite 1976; Jackson and Manelli 1997; Reny and Perry 2006; Pesendorfer and Swinkels 2000; Swinkels 2001; BodohCreed 2010). In the market design literature, many recent papers have focused on large markets (Kojima and Manea 2009; Manea 2009; Budish and Cantillon Forthcoming; Budish Forthcoming; Che and Kojima 2010; Azevedo and Budish 2011).

More closely related to our model, are the contributions in market design that study large matching markets (Roth and Peranson 1999; Immorlica and Mahdian 2005; Kojima and Pathak 2009; Lee 2011). The focus of these papers is quite different from ours. Roth and Peranson (1999), using simulations and data from the National Resident Matching Program observed that, even though stable matching mechanisms are manipulable in theory, they seem to be very close to strategyproof in large markets. This spurred several subsequent papers that theoretically evaluate this proposition. Our work differs from this literature in two key aspects. The first is that previous work has focused on showing approximate incentive compatibility of stable matching mechanisms. $^{12}$ In contrast, we characterize the limit of the set of stable matchings in

\footnotetext{
${ }^{12}$ These papers are also typically concerned with showing that in large matching markets the set of stable matchings is small, according to some definition. These are usually referred to as core convergence results. Indeed, establishing that supply and demand uniquely clear the market in the continuum model is one of our main results, as it adds tractability to the continuum model, and is an important feature of matching markets. Moreover, we prove a core convergence result showing that the set of stable matchings does become small in large economies. Moreover, while our setting is different, we prove this result without the assumptions of short preference lists, or a specific probability generating process for preferences, which is necessary for the combinatorial arguments commonly used in this literature. Another related strand of the literature imposes conditions on preferences to guarantee uniqueness of a stable matching in a given finite economy (Eeckhout 2000; Clark 2006;
} 
large matching markets. Second, the type of limit we take is different. While papers in this literature consider the limit where both sides of the market grow, we consider the case where there is a fixed finite number of colleges, and the number of students grows. The two different limits are suited for analyzing different situations. The former models a situation where each college or firm is a small part of a thick market, and its choices have a vanishing effect on other firms. In contrast, our model is better suited for situations where firms are differentiated, and may have some market power. In such settings, firms do have incentives to misreport preferences to a mechanism, for example by reducing quantity as in a standard oligopoly model (Azevedo 2011).

One paper that does give a sharp asymptotic characterization of the outcome of a mechanism is Che and Kojima (2010). Their main result is that the random serial dictatorship mechanism is asymptotically equivalent to Bogomolnaia and Moulin's (2001) probabilistic serial mechanism. As we discuss below, this is a particular case of our characterization of school choice mechanisms. The derivation of such results is considerably simpler within our framework; instead of employing combinatorial arguments as in the extant literature, we apply differential topology techniques, an approach introduced by Debreu (1970) in general equilibrium theory.

Another central contribution of our paper is the characterization of stable matchings in terms of cutoffs clearing supply and demand. We highlight several related results in the literature. An early result by Roth and Sotomayor (1989) shows that different entering classes in a college at different stable matchings are ordered in the sense that, save for students which are in both entering classes, all students of an entering class are better than those in the other entering class. This suggests that parametrizing the set of stable matchings by the lowest ranked student is possible, though their result does not describe such a parametrization, and the proof is independent from ours. Balinski and Sönmez (1999) give a characterization of fair allocations ${ }^{13}$ based on threshold scores. Biró (2007) studies the algorithm used to compute the outcome of college admissions in Hungary. The algorithm, while very similar to the Gale and Shapley algorithm, uses scores at each stage. Biró (2007) states without proof that a definition of stability in terms of cutoffs is equivalent to the standard definition. Abdulkadiroglu et al. (2008) consider a particular continuum economy, motivated by a school choice problem. They compute a stable matching of this economy, and find that this stable matching can be described in terms of cutoffs.

Niederle and Yariv 2009). Recent work has sought to bound the number of stable matchings given restrictions on preferences (Samet 2010).

${ }^{13}$ Formally, fairness is a concept that applied to the "student placement problem" as defined by Balinski and Sönmez (1999). A matching is individually rational, fair, and non-wasteful if and only if it is stable for the "college admissions problem" that is associated to the original problem, as defined by Balinski and Sönmez (1999). 
Finally, an influential paper by Adachi (2000) gives a characterization of stable matchings in terms of fixed points of an operator over pre-matchings. ${ }^{14}$ This insight has been widely applied in the matching with contracts literature. ${ }^{15}$ Adachi's (2000) result is quite different from ours, as pre-matchings are considerably more complex than cutoffs, and our characterization is in terms of supply and demand and not a fixed point. However, it is informative to dissect the connection. A pre-matching specifies a college for each student, and a set of students for each college. Therefore, the very dimensionality of the set of pre-matchings is considerably larger than that of cutoffs, which specify only an admission threshold for each college. Adachi defines an operator over pre-matchings as follows. Given a pre-matching $v$, agents on either side pick their preferred matches that would be willing to match with them and leave their matches at $v$. He then shows that stable matchings are associated with fixed points of this operator. A commonality of the two characterizations is therefore that pre-matchings work as reservation values for each agent in the Adachi operator. However, Adachi's construction necessitates the extra information included in the high-dimensional object of pre-matchings, and cannot simply be reduced to cutoffs. To clarify this point, in Appendix D we give a simple example where, starting with a pre-matching defined by market clearing cutoffs, Adachi's map produces a pre-matching that does not have the cutoff structure.

Our characterization Lemma 2 is analogous to the Fundamental Theorems of Welfare Economics. Segal (2007) shows that these theorems may be stated for a wide class of social choice problems: namely, socially optimal outcomes can be decentralized with a notion of price equilibrium that is appropriate for the problem. Furthermore, he characterizes the prices that that verify a problem's solutions with minimal communication (Segal's Theorems 2, 3). Applied to stable many-to-one matching, his characterization yields pre-matchings as the appropriate prices (Segal's Proposition 5). In our model, where colleges' preferences are defined by students' scores, the minimally informative prices in Segal's Theorem 3 coincide with our notion of market clearing cutoffs.

Finally, some recent papers explore connections between the matching theoretic concept of stability and Walrasian Equilibrium (Hatfield and Milgrom 2005; Hatfield et al. 2011b), and their equivalence in certain settings. ${ }^{16}$ While we do make such a connection, the scope and spirit of our result is quite different. Our results imply that stable matchings may be found by solving for selectivity cutoffs $P$ that equate demand and supply for each college. This means that stable matchings can be found by solving

\footnotetext{
${ }^{14}$ These ideas have been extended to many-to-one and many-to-many matching markets in important papers by Echenique and Oviedo (2004, 2006).

${ }^{15}$ See for example Ostrovsky (2008); Hatfield and Milgrom (2005); Echenique (Forthcoming).

${ }^{16}$ Specifically,Hatfield et al. (2011b) show that in a trading network with quasilinear utilities, free trasnfers of a numeraire between agents, and substitutable preferences the set of stable outcomes is essentially equivalent to the set of Walrasian equilibria.
} 
market clearing equations for a given excess demand curve $D(P)-S$. This is equivalent to solving for equilibrium prices in a general equilibrium economy. This is a useful connection, and cutoffs do share some properties with prices. However, we highlight that cutoffs are not prices, and there is no equivalence between stability and Walrasian equilibrium in our model.

\section{The Continuum Model}

2.1. Definitions. We begin the exposition with the simpler, and novel continuum model, and examine its connection with the standard discrete Gale and Shapley (1962) model in Sections 3 and 4. The model follows closely the Gale and Shapley (1962) college admissions model. The main departure is that a finite number of colleges $C=\{1,2, \ldots, C\}$ is matched to a continuum mass of students. A student is described by her type $\theta=\left(\succ^{\theta}, e^{\theta}\right)$. $\succ^{\theta}$ is the student's strict preference ordering over colleges. The vector $e^{\theta} \in[0,1]^{C}$ describes the colleges' ordinal preferences for the student. We refer to $e_{c}^{\theta}$ as student $\theta^{\prime}$ 's score at college $c$. Colleges prefer students with higher scores. That is, college $c$ prefers ${ }^{17}$ student $\theta$ over $\theta^{\prime}$ if $e_{c}^{\theta}>e_{c}^{\theta^{\prime}}$. To simplify notation we assume that all students and colleges are acceptable. ${ }^{18}$ Let $\mathcal{R}$ be the set of all strict preference orderings over colleges. We denote the set of all student types by $\Theta=\mathcal{R} \times[0,1]^{C}$.

A continuum economy is given by $E=[\eta, S]$, where $\eta$ is a probability measure ${ }^{19}$ over $\Theta$ and $S=\left(S_{1}, S_{2}, \ldots, S_{C}\right)$ is a vector of strictly positive capacities for each college. We make the following assumption on $\eta$, which corresponds to colleges having strict preferences over students in the discrete model.

Assumption 1. (Strict Preferences) Every college's indifference curves have $\eta$ measure 0 . That is, for any college $c$ and real number $x$ we have $\eta\left(\left\{\theta: e_{c}^{\theta}=x\right\}\right)=0$.

The set of all economies satisfying Assumption 1 is denoted by $\mathcal{E}$.

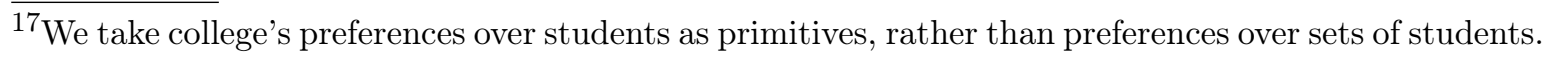
It would have been equivalent to start with preferences over sets of students that were responsive to the preferences over students, as in Roth (1985).

${ }^{18}$ This assumption is without loss of generality. If some students find some schools unacceptable we can generate an equivalent economy where all schools are acceptable. Add a fictitious "unmatched" school with a large capacity and set student preferences to rank it as they would rank being unmatched. Set student preferences to rank all unacceptable schools as acceptable, but ranked below the fictitious school. Since the fictitious school never reaches its capacity, any student that is matched to an unacceptable school can form a blocking pair with the fictitious school. Therefore stable matching of the resulting economy are equivalent to stable matching of the original one. Likewise, we can add a fictitious mass of students that would be ranked bellow all acceptable students and ranked above the unacceptable students.

${ }^{19} \mathrm{We}$ must also specify a $\sigma$-algebra where $\eta$ is defined. The set $\Theta$ is the product of $[0,1]^{C}$ and the finite set of all possible orderings. We take the Borel $\sigma$-algebra of the product topology (the standard topology for $\mathbb{R}^{C}$ times the set of all subsets topology for the discrete set of preference orderings) .
} 
A matching $\mu$ describes an allocation of students to colleges. Formally, a matching in a continuum economy $E=[\eta, S]$ is a measurable function $\mu: C \cup \Theta \rightarrow 2^{\Theta} \cup(C \cup \Theta)$, such that

(1) For all $\theta \in \Theta: \mu(\theta) \in C \cup\{\theta\}$.

(2) For all $c \in C: \mu(c) \subseteq \Theta$, and $\eta(\mu(c)) \leq S_{c}$.

(3) $c=\mu(\theta)$ iff $\theta \in \mu(c)$.

(4) (Right continuity) For any sequence of students $\theta^{k}=\left(\succ, e^{k}\right)$, with $e^{k}$ converging to $e$, and $e_{c}^{k} \geq e_{c}^{k+1} \geq e_{c}$ for all $k, c$, we can find some large $K$ so that $\mu\left(\theta^{k}\right)=$ $\mu(\theta)$ for $k>K$.

The definition of a matching is analogous to that in the discrete model. Conditions 1-3 mirror those in the discrete model. (1) states that each student is matched to a college or to herself. (2) that colleges are matched to sets of students with measure not exceeding its capacity. (3) is a consistency condition, requiring that a college is matched to a student iff the student is matched to the college.

The technical Condition (4) is novel. It states that given a sequence of students $\theta^{k}=\left(e^{k}, \succ\right)$, which are decreasingly desirable, with scores $e^{k} \rightarrow e$, then for large enough $k$ all students $\left(\succ, e^{k}\right)$ in the sequence receive the same allocation, and the limit student $(e, \succ)$ receives this allocation too. The reason why we impose this condition is that in the continuum model it is always possible to add an extra measure 0 set of students to a school without having it exceed its capacity. This would generate multiplicities of stable matchings that differ only in sets of measure 0 . Condition 4 rules out such multiplicities. The intuition is that right continuity implies that a stable matching always allows an extra measure 0 set of students into a college when this can be done without compromising stability. Other than eliminating such multiplicities up to a measure 0 set of students, the condition does not affect the set of stable matchings.

A student-college pair $(\theta, c)$ blocks a matching $\mu$ at economy $E$ if the student $\theta$ prefers $c$ to her match and either (i) college $c$ does not fill its quota or (ii) college $c$ is matched to another student that has a strictly lower score than $\theta$. Formally, $(\theta, c)$ blocks $\mu$ if $c \succ^{\theta} \mu(\theta)$ and either (i) $\eta(\mu(c))<S_{c}$ or (ii) there exists $\theta^{\prime} \in \mu(c)$ with $e_{c}^{\theta^{\prime}}<e_{c}^{\theta}$.

Definition 1. A matching $\mu$ for a continuum economy $E$ is stable if it is not blocked by any student-college pair.

A stable matching always exists. Since the proof is similar to Gale and Shapley's (1962) existence proof in the discrete case, we relegate it to the Appendix (see Corollary A1).

We refer to the stable matching correspondence as the correspondence associating each economy in $\mathcal{E}$ with its set of stable matchings. In some sections in the paper the 
economy is held fixed. Whenever there is no risk of confusion we will omit dependence of certain quantities on the economy.

2.2. The Supply and Demand Characterization of Stable Matchings. In the theory of competitive equilibrium, prices play a key role. In this section we show that selectivity thresholds at each college, which we term cutoffs, play a a similar role in matching markets, with respect to two key dimensions. One important property of prices is decentralizing the allocation. If agents' optimal choices are unique, prices determine the allocation, with each agent simply choosing her favorite bundle that is affordable. Another property is that the task of finding an equilibrium is reduced to finding a vector of prices that clears demand and supply. As the dimensionality of this vector is often small compared to the number of agents in the economy, prices play an important computational role. It is important to highlight that cutoffs are very different than prices, and prices have many properties that cutoffs do not share. For example, in general equilibrium the price ratio of goods gives the marginal rate of substitution of a consumer who buys a positive amount of each. No such relationship for cutoffs holds in our setting, where the allocation depends exclusively on ordinal preferences. Yet, cutoffs and prices do share the two properties outlined above.

Throughout this subsection, we fix an economy $E$, and abuse notation by omitting dependence on $E$ when there is no risk of confusion. A cutoff is a minimal score $P_{c} \in[0,1]$ required for admission at a college $c$. We say that a student $\theta$ can afford college $c$ if $P_{c} \leq e_{c}^{\theta}$, that is $c$ would accept $\theta$. A student's demand given a vector of cutoffs is her favorite college among those that would accept her. That is,

$$
D^{\theta}(P)=\arg \max _{\succ \theta}\left\{c \mid P_{c} \leq e_{c}^{\theta}\right\} \cup\{\theta\} .
$$

Aggregate demand for college $c$ is the mass of students that demand it,

$$
D_{c}(P)=\eta\left(\left\{D^{\theta}(P)=c\right\}\right) .
$$

A market clearing cutoff is a vector of cutoffs that clears supply and demand for colleges.

Definition 2. A vector of cutoffs $P$ is a market clearing cutoff if it satisfies the following market clearing equations: for all $c$

$$
D_{c}(P) \leq S_{c}
$$

and $D_{c}(P)=S_{c}$ if $P_{c}>0$.

There is a natural one-to-one correspondence between stable matchings and market clearing cutoffs. To describe this correspondence, we define two operators. Given a market clearing cutoff $P$, we define the associated matching $\mu=\mathcal{M P}$ using the demand 
function:

$$
\mu(\theta)=D^{\theta}(P)
$$

Conversely, for a stable matching $\mu$, we define the associated cutoff $P=\mathcal{P} \mu$ by:

$$
P_{c}=\inf _{\theta \in \mu(c)} e_{c}^{\theta}
$$

The operators $\mathcal{M}$ and $\mathcal{P}$ give a bijection between stable matchings and market clearing cutoffs.

Lemma 1. (Supply and Demand Lemma) If $\mu$ is stable matching, then $\mathcal{P} \mu$ is a market clearing cutoff. If $P$ is a market clearing cutoff, then $\mathcal{M} P$ is a stable matching. In addition, the operators $\mathcal{P}$ and $\mathcal{M}$ are inverses of each other.

The Lemma subsumes two useful facts. First, stable matchings all have a very special structure. Given any stable matching $\mu$, there must exist a corresponding vector of cutoffs such that each student is matched to $\mu(\theta)=D^{\theta}(P)$. Therefore, any stable matching corresponds to each student choosing her favorite college conditional on being accepted at a vector of cutoffs $P$. Therefore, if one is interested in stable matchings, it is not necessary to consider all possible matchings, but only those that have this very special structure. This is similar to the decentralization property that prices have in competitive equilibrium, where each agent's allocation is determined by her preferences, endowment, and market prices. ${ }^{20}$

Second, the Lemma implies that computing stable matchings is equivalent to finding market clearing cutoffs, as $\mathcal{M}$ and $\mathcal{P}$ are a one-to-one correspondence between these two sets. Therefore, stable matchings can be found by solving market clearing equations, balancing demand $D(P)$ and supply $S$. In particular, finding stable matchings is equivalent to finding competitive equilibria of an economy with quasilinear preferences and aggregate demand function $D(P)$.

\subsection{Example: The Supply and Demand Characterization and Convergence of Discrete Economies to Continuum Economies. This simple example illus-} trates the supply and demand characterization of stable matchings and previews our convergence results. There are two colleges $c=1,2$, and the distribution of students $\eta$ is uniform. That is, there is a mass $1 / 2$ of students with each preference list 1,2 or 2,1 , and each mass has scores distributed uniformly over $[0,1]^{2}$ (Figure 1 ). If both colleges had capacity $1 / 2$, the unique stable matching would have each student matched to her favorite college. To make the example interesting, assume $S_{1}=1 / 4, S_{2}=1 / 2$.

\footnotetext{
${ }^{20} P$ decentralizes the allocation in the sense that, as in competitive equilibrium, the allocation is determined solely by $\left(\succ^{\theta}, e^{\theta}\right)$ and $P$. Thus, $P$ summarizes the effect of aggregate market conditions on $\theta$ 's allocation. Note, however, that $e^{\theta}$ represents colleges' preferences. Therefore, if colleges have private information over their preferences, it is not true that $P$ decentralizes the allocation in the sense that a student's allocation only depends on her private information and $P$. See also footnote ??.
} 


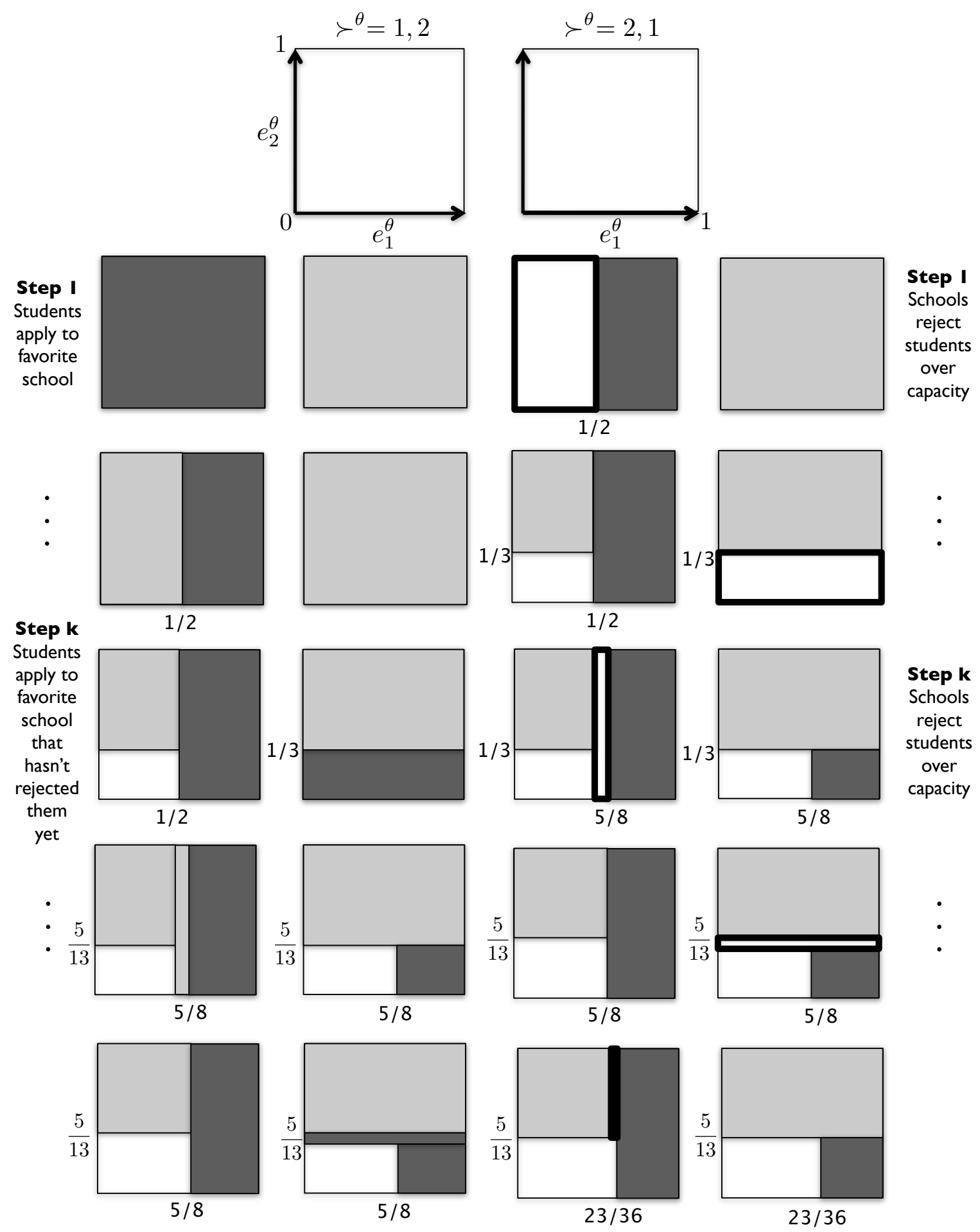

Figure 1. The set of student types $\Theta$ is represented by the two squares on the top panel. The left square represents students that prefer college 1 , and the right square students who prefer college 2. Scores at each college are represented by the $(x, y)$ coordinates. The lower panels show the first 10 steps of the Gale-Shapley student-proposing algorithm. In each line, students apply to their favorite colleges that have not yet rejected them in the left panel, and colleges reject students to remain within capacity in the right panel. Students in dark gray are tentatively assigned to college 1 , and in light gray tentatively assigned to college 2 . 
A familiar way of finding stable matchings is using the student-proposing deferred acceptance algorithm. In the Appendix, we formally define the algorithm, and prove that it converges to a stable matching. Here, we informally follow the algorithm for this example, to build intuition on the special structure of stable matchings. At each step of the algorithm unassigned students propose to their favorite college out of the ones that still haven't rejected them. If a college has more students than its capacity assigned to it, it rejects the lower ranked students currently assigned, to stay within its capacity. Figure 1 displays the trace of the algorithm in our example. In the first step, all students apply to their favorite college. Because college 1 only has capacity $1 / 4$, and each square has mass $1 / 2$, it then rejects half of the students who applied. The rejected students then apply to their second choice, college 2 . But this leaves college 2 with $1 / 2+1 / 4=3 / 4$ students assigned to it, which is more than its quota. College 2 then rejects its lower ranked students. Those who had already been rejected stay unmatched. But those who hadn't been rejected by college 1 apply to it, leaving it with more students than capacity, and the process continues. Although the algorithm does not reach a stable matching in a finite number of steps, it always converges, and its pointwise limit (shown in Figure 2) is a stable matching (this is proven in Appendix A). Figure 1 hints at this, as the measure of students rejected in each round is becoming smaller and smaller.

However, Figures 1 and 2 give much more information than simply convergence of the deferred acceptance mechanism. We can see that cutoffs yield a simpler decentralized way to describe the matching. Note that all students accepted to college 1 have a score $e_{1}^{\theta}$ above a cutoff of $P_{1} \approx .640$, and those accepted to college 2 have a score $e_{2}^{\theta}$ above some cutoff $P_{2} \approx .390$. Hence, had we known these numbers in advance, it would have been unnecessary to run the deferred acceptance algorithm. All we would have to do is assign each student to her favorite college such that her score is above the cutoff, $e_{c}^{\theta} \geq P_{c}$ (Supply and Demand Lemma 1).

Additionally, the Supply and Demand Lemma gives another way of finding stable matchings. Instead of following the deferred acceptance algorithm, one may simply look for cutoffs that equate supply and demand $D(P)=S$. Consider first demand for college 1. The fraction of students in the left square of Figure 2 demanding college 1 is $1-P_{1}$. And in the right square it is $P_{2}\left(1-P_{1}\right)$. Therefore $D_{1}(P)=\left(1+P_{2}\right)\left(1-P_{1}\right) / 2$. $D_{2}$ has an analogous formula, and the market clearing equations are

$$
\begin{aligned}
& S_{1}=1 / 4=\left(1+P_{2}\right)\left(1-P_{1}\right) / 2 \\
& S_{2}=1 / 2=\left(1+P_{1}\right)\left(1-P_{2}\right) / 2 .
\end{aligned}
$$



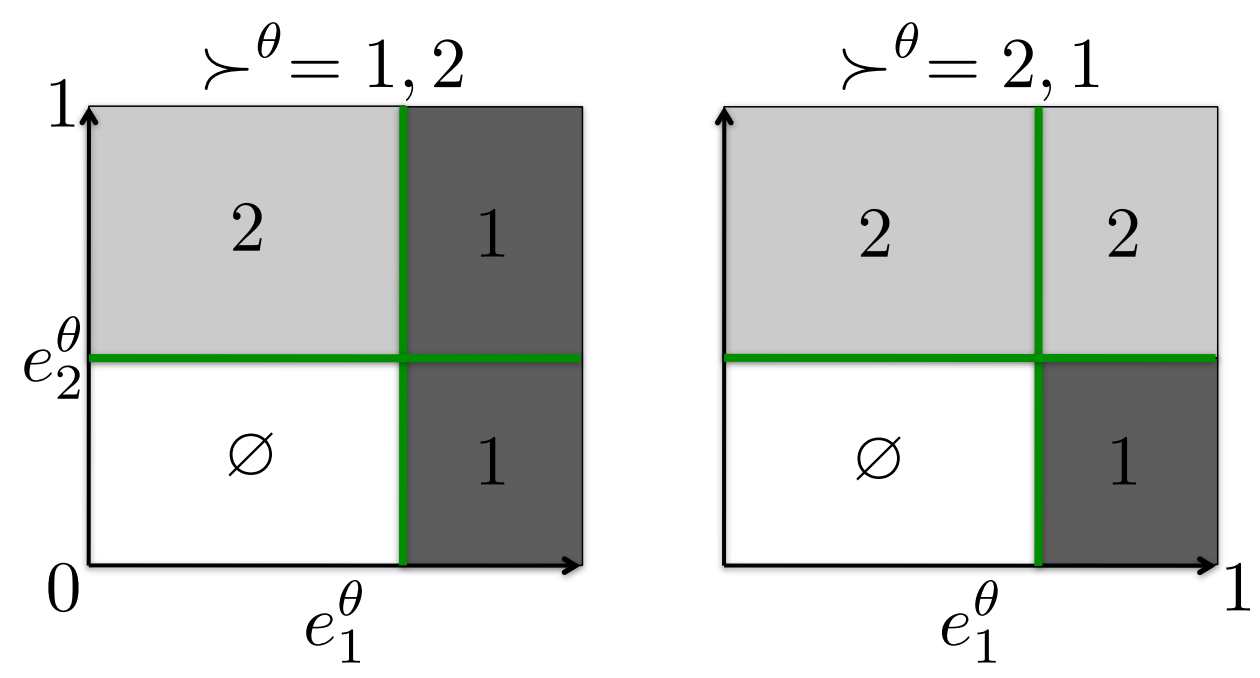

Figure 2. A stable matching in a continuum economy with two colleges. The two squares represent the set of student types $\Theta$. The left square represents students that prefer college 1, and the right square students who prefer college 2 . Scores at each college are represented by the $(x, y)$ coordinates. The white area represents unmatched students, dark gray are matched to college 1 , and light gray to college 2.

Solving this system, we get $P_{1}=(\sqrt{17}+1) / 8$ and $P_{2}=(\sqrt{17}-1) / 8$. In particular, because the market clearing equations have a unique solution, the economy has a unique stable matching (Theorem 1 shows this is a much more general phenomenon).

We show below that the cutoff characterization is also valid in the discrete Gale and Shapley (1962) model, save for the fact that in discrete model each stable matching may correspond to more than one market clearing cutoff (Lemma 2). Figure 3 illustrates cutoffs for a stable matching in a discrete economy with 1,000 students. The 1,000 students were assigned random types, drawn from the distribution $\eta$ used in the continuum example. For that reason, the empirical distribution of types of the discrete economy approximates that of the continuum economy. In this sense, this discrete economy approximates the continuum economy. Note that the cutoffs in the discrete economy are approximately the same as the cutoffs in the continuum economy. Theorem 2 shows that, generically, the market clearing cutoffs of approximating discrete economies approach market clearing cutoffs of the continuum economy.

\section{The Discrete Model And Convergence Notions}

This section reviews the discrete Gale and Shapley (1962) college admissions model, states the discrete Supply and Demand Lemma, and defines convergence notions used to state our main results. 

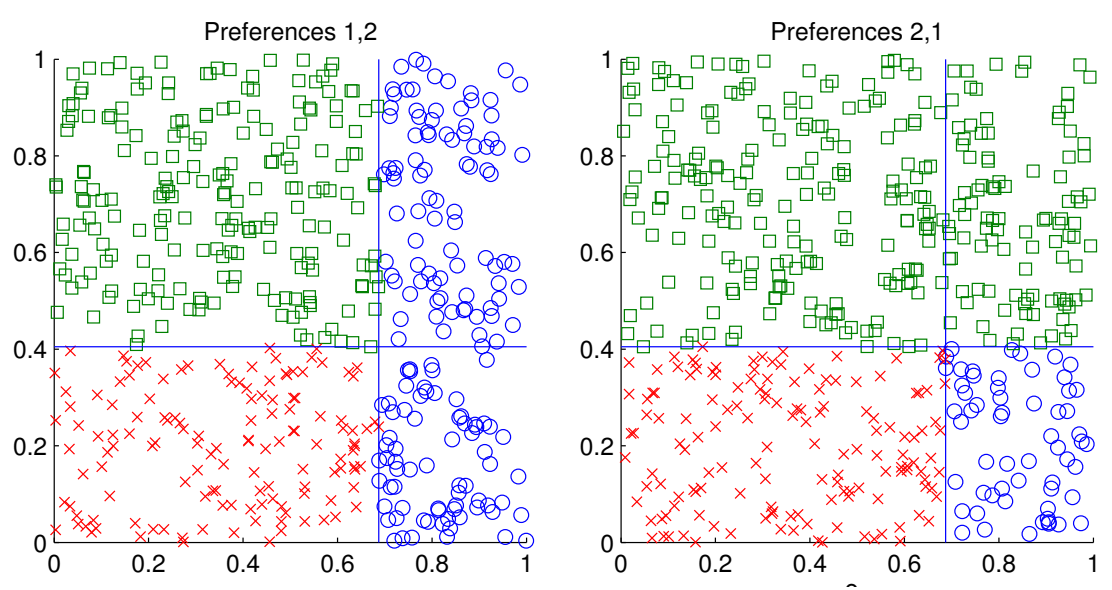

Figure 3. Cutoffs of a stable matching in a discrete economy approximating the continuum economy in the example. The two squares represent the set of student types $\Theta$. The left square represents students prefer college 1, and the right square students who prefer college 2. Scores at each college are represented by the $(x, y)$ coordinates. There are 2 colleges, with capacities $q_{1}=250, q_{2}=500.500$ students have preferences $\succ^{\theta}=1,2, \emptyset$ and 500 students have preferences $2,1, \emptyset$. Scores $e^{\theta}$ were drawn independently according to the uniform distribution in $[0,1]^{2}$. The Figure depicts the student-optimal stable matching. Balls represent students matched to college 1 , squares to college 2, and Xs represent unmatched students.

3.1. The Discrete Gale and Shapley Model. The set of colleges is again $C$. A finite economy $F=[\tilde{\Theta}, \tilde{S}]$ specifies a finite set of students $\tilde{\Theta} \subset \Theta$, and an integer vector of capacities $\tilde{S}_{c}>0$ for each college. We assume that no college is indifferent between two students in $\tilde{\Theta}$. A matching for finite economy $F$ is a function $\tilde{\mu}: C \cup \tilde{\Theta} \rightarrow 2^{\tilde{\Theta}} \cup(C \cup \tilde{\Theta})$ such that

(1) For all $\theta$ in $\tilde{\Theta}: \mu(\theta) \in C \cup\{\theta\}$.

(2) For all $c \in C: \mu(c) \in 2^{\tilde{\Theta}}$ and $\# \mu(c) \leq \tilde{S}_{c}$.

(3) For all $\theta \in \tilde{\Theta}, c \in C: \mu(\theta)=c$ iff $\theta \in \mu(c)$.

These conditions may be interpreted as follows. (1) Each student is matched to a college or to herself, (2) each college is matched to at most $\tilde{S}_{c}$ students, and (3) the consistency condition that a college is matched to a student iff the student is matched to the college.

The definition of a blocking pair is the same as in Section 2.1. A matching $\tilde{\mu}$ is said to be stable for finite economy $F$ if it has no blocking pairs.

Given a finite economy $F=[\tilde{\Theta}, \tilde{S}]$, we may associate with it the empirical distribution of types

$$
\eta=\sum_{\theta \in \tilde{\Theta}} \frac{1}{\# \tilde{\Theta}} \delta_{\theta},
$$


where $\delta_{x}$ denotes is the probability distribution associating probability 1 to the point $x$. The supply of seats per student is given by $S=\tilde{S} / \# \tilde{\Theta}$. Note that $[\eta, S]$ uniquely determine a discrete economy $F=[\tilde{\Theta}, S]$, as $\tilde{\Theta}=\operatorname{support}(\eta)$ and $\tilde{S}=S \cdot \# \tilde{\Theta}$. Therefore, either pair $[\tilde{\Theta}, \tilde{S}]$ or $[\eta, S]$ uniquely determine a finite economy $F$. Throughout the remainder of the text we will abuse notation and refer to finite economies simply as

$$
F=[\eta, S] .
$$

This convention will be useful for stating our convergence results below, as it makes finite economies $F$ comparable to continuum economies $E$.

3.1.1. Cutoffs. In this section we fix a finite economy $F=[\eta, S]$, and will omit dependence on $F$ in the notation. A cutoff is a number $P_{c}$ in $[0,1]$ specifying an admission threshold for college $c$. Given a vector of cutoffs $P$, a student's individual demand $D^{\theta}(P)$, the aggregate demand function $D(P)$, and market clearing cutoffs are defined as in Section 2.2.

In the discrete model, we define the operators $\tilde{\mathcal{M}}$ and $\tilde{\mathcal{P}}$, which have essentially the same definitions as $\mathcal{M}$ and $\mathcal{P}$. Given market clearing cutoffs $P, \tilde{\mu}=\tilde{\mathcal{M}} P$ is the matching such that for all $\theta \in \tilde{\Theta}: \tilde{\mu}(\theta)=D^{\theta}(P)$. Given a stable matching $\tilde{\mu}, P=\tilde{\mathcal{P}} \tilde{\mu}$ is given by $P_{c}=0$ if $\eta(\tilde{\mu}(c))<S_{c}$ and $P_{c}=\min _{\theta \in \tilde{\mu}(c)} e_{c}^{\theta}$ otherwise.

In the discrete case, we have the following analogue of the Supply and Demand Lemma.

Lemma 2. (Discrete Supply and Demand Lemma) In a discrete economy, the operators $\tilde{\mathcal{M}}$ and $\tilde{\mathcal{P}}$ take stable matchings into market clearing cutoffs, and vice versa. Moreover, $\tilde{\mathcal{M}} \tilde{\mathcal{P}}$ is the identity.

Proof. Consider a stable matching $\tilde{\mu}$, and let $P=\tilde{\mathcal{P}} \tilde{\mu}$. Any student $\theta$ who is matched to a college $c=\tilde{\mu}(\theta)$ can afford her match, as $P_{c} \leq e_{c}^{\theta}$ by the definition of $\tilde{\mathcal{P}}$. Likewise, students who are unmatched may always afford being unmatched. Note that no student can afford a college $c^{\prime} \succ^{\theta} \tilde{\mu}(\theta)$ : if she could, then $P_{c^{\prime}} \leq e_{c^{\prime}}^{\theta}$, and by the definition of $\tilde{\mathcal{P}}$, there would be another student $\theta^{\prime}$ matched to $c^{\prime}$ with $e_{c^{\prime}}^{\theta^{\prime}}<e_{c^{\prime}}^{\theta}$, or empty seats at $c^{\prime}$, which would contradict $\tilde{\mu}$ being stable. Consequently, no student can afford any option better than $\tilde{\mu}(\theta)$, and all students can afford their own match $\tilde{\mu}(\theta)$. This implies $D^{\theta}(P)=\tilde{\mu}(\theta)$. This proves both that $\tilde{\mathcal{M}} \tilde{\mathcal{P}}$ is the identity, and that $P$ is a market clearing cutoff.

In the other direction, let $P$ be a market clearing cutoff, and $\tilde{\mu}=\tilde{\mathcal{M}} P$. By the definition of the operator and the market clearing conditions it is a matching, so we only have to show there are no blocking pairs. Assume by contradiction that $(\theta, c)$ is a blocking pair. If $c$ has empty slots, then $P_{c}=0 \leq e_{c}^{\theta}$. If $c$ is matched to a student $\theta^{\prime}$ that 
it likes less than $\theta$, then $P_{c} \leq e_{c}^{\theta^{\prime}} \leq e_{c}^{\theta}$. Hence, we must have $P_{c} \leq e_{c}^{\theta}$. But then we have $c \preceq^{\theta} D^{\theta}(P)=\tilde{\mu}(\theta)$, so $(\theta, c)$ cannot be a blocking pair, reaching a contradiction.

The Lemma guarantees that stable matchings always have a cutoff structure $\tilde{\mu}=$ $\tilde{\mathcal{M}} P$ even in a discrete market. Therefore, it is still true that in the discrete model cutoffs decentralize the allocation, and that for finding all stable matchings one only has to consider market clearing cutoffs. The only difference between the Lemmas for the discrete and continuous case, is that in the continuum model the correspondence between market clearing cutoffs and stable matchings is one-to-one. In the discrete model, in contrast, each stable matching may be associated with several market clearing cutoffs. The reason is that changing a particular $P_{c}$ in a range where there are no students with scores $e_{c}^{\theta}$ does not affect the demand function.

3.2. Convergence Notions. To describe our convergence results, we must define notions of convergence for economies and stable matchings. We will say that a sequence of continuum economies $\left\{E^{k}\right\}_{k \in \mathbb{N}}, E^{k}=\left[\eta^{k}, S^{k}\right]$ converges to a limit economy $E=[\eta, S]$ if the measures $\eta^{k}$ converge in the weak sense to $\eta,{ }^{21}$ and if the vectors $S^{k}$ converge to $S$.

We take the distance between stable matchings to be the distance between their associated cutoffs in the supremum norm in $\mathbb{R}^{C}$. That is, the distance between two stable matchings $\mu$ and $\mu^{\prime}$ is

$$
d\left(\mu, \mu^{\prime}\right)=\left\|\mathcal{P} \mu-\mathcal{P} \mu^{\prime}\right\|_{\infty} .
$$

A sequence of finite economies $\left\{F^{k}\right\}_{k \in \mathbb{N}}, F^{k}=\left[\eta^{k}, S^{k}\right]$ converges to a continuum economy $E=[\eta, S]$ if the empirical distribution of types $\eta^{k}$ converges to $\eta$ in the weak sense, and the vectors of capacity per student $S^{k}$ converge to $S$.

Given a stable matching of a continuum economy $\mu$, and a stable matching of a finite economy $\tilde{\mu}$, we define

$$
d(\tilde{\mu}, \mu)=\sup _{P}\|P-\mathcal{P} \mu\|_{\infty}
$$

over all vectors $P$ with $\tilde{\mathcal{M}} P=\tilde{\mu}$.

The sequence of stable matchings $\left\{\tilde{\mu}^{k}\right\}_{k \in \mathbb{N}}$ with respect to finite economies $F$ converges to stable matching $\mu$ of continuum economy $E$ if $d\left(\tilde{\mu}^{k}, \mu\right)$ converges to 0 .

Finally, we will show that the set of stable matchings of large finite economies becomes small under certain conditions. To state this, we define the diameter of the set of stable matchings of a finite economy $F$ as $\sup \left\{\left\|P-P^{\prime}\right\|_{\infty}: P\right.$ and $P^{\prime}$ are market clearing cutoffs of $F$ \}.

\footnotetext{
${ }^{21}$ Weak convergence of measures is defined as the integrals $\int f d \eta^{k}$ converging to $\int f d \eta$ for every bounded continuous function $f: \Theta \rightarrow \mathbb{R}$. In analysis, this is usually termed weak-* convergence.
} 


\section{Main Results: Convergence and Uniqueness}

We are now ready to state the main results of the paper. The first result shows that, typically, continuum economies have a unique stable matching. We first define a minimal notion of smoothness of measures.

Definition 3. Measure $\eta$ is regular if the closure of the set of points

$$
\left\{P \in[0,1]^{C}: D(\cdot \mid \eta) \text { is not continuosuly differentiable at } P\right\}
$$

and its image under $D(\cdot \mid \eta)$ have Lebesgue measure 0.

This definition is very general, and includes cases where $\eta$ does not have a density, and where it has points where demand is not differentiable. While the condition is somewhat technical, it is always satisfied for example if $D(\cdot \mid \eta)$ is continuously differentiable, and also if $\eta$ admits a continuous density.

The next result gives conditions for the continuum model to have a unique stable matching.

Theorem 1. Consider an economy $E=[\eta, S]$.

(1) If $\eta$ has full support, then $E$ has a unique stable matching.

(2) If $\eta$ is any regular distribution, then for almost every vector of capacities $S$ with $\sum_{c} S_{c}<1$ the economy $E$ has a unique stable matching.

The Theorem has two parts. First, it shows that whenever $\eta$ has full support, a limit economy has a unique stable matching. Therefore, whenever the set of students is rich enough, an economy has a unique stable matching. ${ }^{22}$ Moreover, it shows that, even if the full support assumption does not hold, in a very general setting for almost every $S$ there exists a unique stable matching. Therefore, the typical case is for the continuum model to have a unique stable matching, with supply and demand uniquely clearing the market.

Proof Sketch. Here we outline the main ideas in the proof, which is deferred to Appendix B. The core of the argument employs tools from differentiable topology, an approach pioneered in general equilibrium theory by the classic paper of Debreu (1970).$^{23}$ Moreover, the proof uses two results, developed in Appendix A, extending classic results of matching theory to the continuum model. The first is the Lattice Theorem, which

${ }^{22}$ The assumption of full support may be weakened to all sets of the form

$$
\left\{\theta \in \Theta: \theta \nless P, \theta<P^{\prime} \text {, for } P \leq P^{\prime}, P \neq P^{\prime}\right\}
$$

having positive $\eta$ measure. For details see the working paper version of Azevedo (2011). This assumption is satisfied for example in the case of perfectly correlated college preferences, that is when for all $\theta \in \operatorname{support}(\theta)$ and $c, c^{\prime} \in C e_{c}^{\theta}=e_{c^{\prime}}^{\theta}$.

${ }^{23}$ For an overview of the literature spurred by this seminal paper see Mas-Colell (1990). 


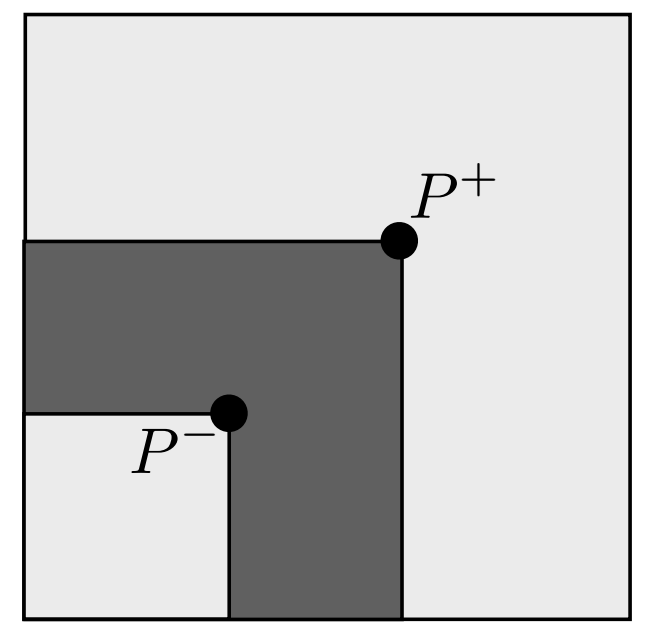

Figure 4. The Figure illustrates the Proof of Theorem 1. The shaded area corresponds scores in the set $\left\{e^{\theta} \in[0,1]^{C}: e^{\theta}<P^{+}, e^{\theta} \nless P^{-}\right\}$, which is used in the proof of Theorem 1 Part (1). Students who find all schools acceptable and have scores in this set are matched under $P^{-}$but are unmatched under $P^{+}$.

guarantees that for any economy $E$ the set of market clearing cutoffs is a complete lattice. In particular, this implies that there exist smallest and largest vectors of market clearing cutoffs. In the proof we will denote these cutoffs $P^{-}$and $P^{+}$. The other result is the Rural Hospitals Theorem, which guarantees that the measure of unmatched students in any two stable matchings is the same.

Part (1).

In the text we focus on the case where $P^{+}>0$, and defer the general case to the Appendix. Note that the set of unmatched students at $P^{+}$contains the set of unmatched students at $P^{-}$, and their difference contains the set

$$
\left\{\theta \in \Theta: e^{\theta}<P^{+}, e^{\theta} \nless P^{-}\right\} .
$$

By the Rural Hospitals Theorem, the mass of unmatched agents at $P^{+}$and $P^{-}$must be the same, and therefore this set must have $\eta$ measure 0 . Since $\eta$ has full support, this implies that $P^{-}=P^{+}$, and therefore there is a unique stable matching (Figure 4).

\section{Part (2).}

For simplicity, consider the case where the function $D(P \mid \eta)$ is continuously differentiable. The general case is covered in the Appendix.

We begin by applying Sard's Theorem. ${ }^{24}$ The Theorem states that, given a continuously differentiable function $f: \mathbb{R}^{n} \rightarrow \mathbb{R}^{n}$, we have that for almost every $S_{0} \in \mathbb{R}^{n}$ the derivative $\partial f\left(P_{0}\right)$ is nonsingular at every solution $P_{0}$ of $f\left(P_{0}\right)-S_{0}=0$. The intuition

$\overline{{ }^{24} \text { See Guillemin }}$ and Pollack (1974); Milnor (1997). 
for this result is easy to see in one dimension. It says that if we randomly perturb the graph of a function with a small vertical translation, all roots will have a non-zero derivative with probability 1.

Given $S$, as we assumed that there is excess demand for colleges $\left(\sum_{c} S_{c}<1\right)$, the market clearing cutoffs are the set of roots $P$ of the equation

$$
D(P \mid \eta)=S .
$$

By Sard's Theorem, we have that for almost every $S$, the derivative $\partial_{P} D(\cdot \mid \eta)$ is invertible at every market clearing cutoff associated with $[\eta, S]$. Henceforth, we will restrict attention to an economy $E=[\eta, S]$ where this is the case, as this is the case for almost every $S$.

To reach a contradiction, we assume that $E$ has more than one stable matching. Therefore, there must be smallest and largest market clearing cutoffs with $P^{-} \leq P^{+}$. For simplicity we restrict attention to the case where $0<P^{-}<P^{+}$, and defer the general case to the Appendix. For any $P$ in the cube $\left[P^{-}, P^{+}\right]$, the measure of unmatched students

$$
1-\sum_{c} D_{c}(P \mid \eta)
$$

must be higher than the measure of unmatched students at $P^{-}$but lower than the measure at $P^{+}$. However, by the Rural Hospitals Theorem, this measure must be the same at $P^{-}$and $P^{+}$. Therefore, the expression in equation 4.1 must be constant in the cube $\left[P^{-}, P^{+}\right]$. This implies that the derivative matrix of $D$ at $P^{-}$must satisfy

$$
\sum_{c} \partial D_{c}\left(P^{-} \mid \eta\right)=0
$$

This implies that the matrix of derivatives $\partial D\left(P^{-} \mid \eta\right)$ is not invertible, contradicting Sard's Theorem.

The next Theorem establishes the link between the continuum model and the standard discrete Gale and Shapley model. It shows that when an economy $E$ has a unique stable matching, which is the generic case, (1) it corresponds to the limit of stable matchings of approximating finite economies, (2) approximating finite economies have a small set of stable matchings, and (3) the unique stable matching varies continuously with the fundamentals of the economy.

Theorem 2. Assume that the continuum economy $E$ admits a unique stable matching $\mu$. We then have

(1) For any sequence of stable matchings $\left\{\tilde{\mu}^{k}\right\}_{k \in \mathbb{N}}$ of finite economies in a sequence $\left\{F^{k}\right\}_{k \in \mathbb{N}}$ converging to $E$, we have that $\tilde{\mu}^{k}$ converges to $\mu$. 
(2) Moreover the diameter of the set of stable matchings of $\left\{F^{k}\right\}_{k \in \mathbb{N}}$ converges to 0 .

(3) The stable matching correspondence is continuous at E within the set of continuum economies $\mathcal{E}$.

The Theorem considers continuum economies $E$ with a unique stable matching, which Theorem 1 guarantees that this is the typical case.

Part (1) states that the unique stable matching of the limit economy is the limit of any sequence of stable matchings of approximating finite economies. This implies that the continuum model we propose does correspond to the limit of the discrete Gale and Shapley (1962) model. We emphasize that for a sequence of finite economies $\left\{F^{k}\right\}_{k \in \mathbb{N}}$ to converge to a limit economy $E$ means that the empirical distribution of student types converges, and that the vector of capacities per student converges. Therefore, the economies $F^{k}$ have an increasing number of students, and a fixed number of colleges.

Part (2) states that the diameter of the set of stable matchings of any such sequence of approximating finite economies converges to 0 . This means that, as economies in the sequence become sufficiently large, the set of stable matchings becomes small. More precisely, even if such an economy has several stable matchings, cutoffs are very similar in any stable matching. To a first approximation, supply and demand clear large matching markets uniquely.

Finally, Part (3) states that the stable matching of $E$ varies continuously in the set of all continuum economies $\mathcal{E}$. This means that stable matchings vary continuously with the fundamentals of the economy $E$. This result is of significance for studies that use data and simulations to inform market design (Abdulkadiroglu et al. 2009; Budish and Cantillon Forthcoming). It implies that in large matching markets the conclusions of such simulations are not sensitive to small changes in the parameters.

The proof of Theorem 2 Part (1) is based on the observation that if a sequence of economies $F^{k}$ converges to an economy $E$, then its demand functions converge. With this observation it is possible to prove that any sequence of stable matchings of $\left\{F^{k}\right\}_{k \in \mathbb{N}}$ converges to a stable matching of $E$. Since $E$ has a unique stable matching, any such sequence must converge to the same stable matching. Part (2) then follows from Part (1). As for Part (3), the argument is similar to that in Part (1). Uniqueness plays an important role in Theorem 2. In Section 5.5 we give a knife edge example of an economy with multiple stable matchings, and where Theorem 2 fails. Moreover, we give a result showing that in a large set of cases where uniqueness fails, the set of stable matchings may change discontinuously with small changes in the fundamentals, and that none of the conclusions of Theorem 2 hold. 


\section{Applications and Extensions}

\subsection{Comparative Statics and a Price-Theoretic Analysis of the Incentives to}

Invest in School Quality. To illustrate the derivation of comparative statics in the continuum framework, we will derive measures of the incentives for schools to invest in quality in a city where there is school choice, and schools compete for the best students. This problem is studied in Hatfield et al. (2011a), using the standard discrete Gale and Shapley framework. ${ }^{25}$ They show that in large markets the incentives for schools to invest in quality are nonnegative, but are silent about their magnitude, and to what types of investments schools pursue. To address these issues, we approach the problem from a price-theoretic perspective.

Consider a city with a number of public schools $c=1, \cdots, C$, each with capacity $S_{c}$. Students are assigned to schools through a clearinghouse, via a stable matching mechanism. This is a stark description of the institutional arrangements in New York City. Students are denoted as $i \in I$. Schools' preferences over students are given by scores $e_{c}^{i}$. We assume that the vectors $e^{i}$ are distributed according to a distribution function $G(\cdot)$ in $\mathbb{R}^{C}$, with a continuous density $g>0$.

Students' preferences depend on the quality $\delta_{c} \in \mathbb{R}$ of each school. $\delta_{c}$ should be interpreted as a vertical quality measure, in that all students prefer higher $\delta_{c}$. However, different students may be affected differently by $\delta_{c}$. So, for example, if $\delta_{c}$ measures the quality of a school's calculus course, then students of high academic caliber, or with a focus in math, will be more sensitive to changes in $\delta_{c}$. One of the advantages of our approach is that it predicts which groups of students a school would like to target with improvements in quality. Student $i$ has utility $u_{c}^{i}\left(\delta_{c}\right)>0$ of attending school $c$, increasing in $\delta_{c}$, and utility 0 of being unmatched. The measure of students who are indifferent between two schools is 0 . Given $\delta$, preferences induce a distribution $\eta_{\delta}$ over student types $\Theta$, which we assume to be have a density $f_{\delta}>0$, smooth in $\delta$ and $\theta$.

Under these assumptions, given $\delta$, there exists a unique stable matching $\mu_{\delta}$. Let $P^{*}(\delta)$ be the unique associated market clearing cutoffs. Dependence on $\delta$ will be omitted when there is no risk of confusion.

For concreteness, we define the aggregate quality of a school's entering class as

$$
Q_{c}(\delta)=\int_{\mu_{\delta}(c)} e_{c}^{\theta} d \eta_{\delta}(\theta)
$$

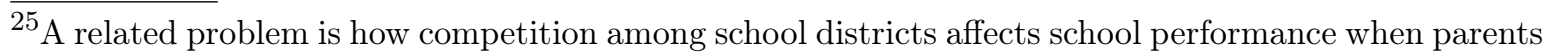
may only choose schools insofar as they can move (Tiebout 1956; Hoxby 2000, 2003). In what follows we follow Hatfield et al. (2011a) and abstract away from the possibility that students can move, and consider the absence of school choice as a benchmark where the incentives derived below are 0. A more realistic approach would be to explicitly model Tiebout competition.
} 
That is, the integral of scores $e_{c}^{\theta}$ over all students matched to the school. We consider how a school's quality affects the quality of its entering class. The motivation is that, following Hatfield et al. (2011a), if schools are concerned about $Q_{c}{ }^{26}$ then a direct link between $\delta_{c}$ an $Q_{c}$ gives school administrators incentives to improve quality $\delta_{c}$.

To derive an expression for the effect of investing in quality, we must define relevant quantities. Let $Y_{c}(P, \delta, e)$ be the fraction of students of scores $e$ who prefer school $c$ out of all schools they can afford.

$$
Y_{c}(P, \delta, e)=\operatorname{Pr}\left\{c \succeq^{\theta} c^{\prime} \forall c^{\prime}: P_{c^{\prime}} \leq e_{c^{\prime}}^{\theta} \mid e^{\theta}=e, \theta \sim \eta_{\delta}\right\},
$$

if $e_{c} \geq P_{c}$ and 0 otherwise. Define the student types who are marginally accepted to school $c^{\prime}$ and would go to school $c$ otherwise as

$$
\tilde{M}_{c^{\prime} c}=\left\{\theta: c^{\prime} \succ^{\theta} c, P_{c^{\prime}}=e_{c^{\prime}}^{\theta}, P_{c} \leq e_{c}^{\theta}, P_{c^{\prime \prime}}>e_{c^{\prime \prime}}^{\theta} \forall c^{\prime \prime} \neq c^{\prime}: c^{\prime \prime} \succ^{\theta} c\right\} .
$$

Let the $C-1$ dimensional mass, and average score of these students be

$$
\begin{aligned}
M_{c^{\prime} c} & =\int_{\tilde{M}_{c^{\prime} c}} f_{\delta}(\theta) d \theta \\
\bar{P}_{c^{\prime} c} & =E\left[e_{c}^{\theta} \mid \theta \in \tilde{M}_{c^{\prime} c}\right] .
\end{aligned}
$$

The effect of school quality $\delta_{c}$ on the quality of the entering class $Q_{c}$ is as follows.

Proposition 1. Assume that $P^{*}(\delta)>0$, and that $P$ is differentiable in $\delta_{c}$. Then $Q_{c}$ is differentiable in $\delta_{c}$, and its derivative can be decomposed as

$$
\frac{d Q_{c}}{d \delta_{c}}=\underbrace{\int_{e: e_{c} \geq P_{c}^{*}}\left[e_{c}-P_{c}^{*}\right] \cdot \frac{d Y_{c}}{d \delta_{c}}\left(P^{*}, \delta, e\right) d G(e)}_{\text {Direct Effect }}-\underbrace{\sum_{c^{\prime} \neq c}\left[\bar{P}_{c^{\prime} c}-P_{c}^{*}\right] \cdot M_{c^{\prime} c} \cdot\left(-\frac{d P_{c^{\prime}}^{*}}{d \delta_{c}}\right)}_{\text {Market Power Effect }} .
$$

The direct effect term is weakly positive, always giving incentives to invest in quality. The market power terms increase (decrease) the incentives to invest in quality if an increase (decrease) in the quality of school c increases the market clearing cutoff of school $c^{\prime}$, that is $d P_{c^{\prime}}^{*}(\delta) / d \delta_{c}>0(<0)$.

The Proposition states that the effect of an increase in quality can be decomposed in two terms. The direct effect is the increase in quality, holding cutoffs $P$ fixed, due to students with $e_{c} \geq P_{c}^{*}$ choosing school $c$ with higher frequency. Note that this integrand is proportional to $e_{c}-P_{c}^{*}$. Since the total number of students that the school takes in is fixed at $S_{c}$, the gain is only a change in composition. As the school attracts more students with scores $e_{c}$ it must shed marginal students with scores $P_{c}^{*}$. The change in

\footnotetext{
${ }^{26}$ There is evidence that schools in NYC are concerned about the quality of their incoming classes, as many schools used to withhold capacity to game the allocation system used previously to the Abdulkadiroglu et al. (2005b) redesign of the match.
} 
quality $e_{c}-P_{c}^{*}$ must be multiplied by the $d Y_{c} / d \delta_{c}$ term, which measures the number of students who change their choices.

The market power effect measures how much the school loses due to its higher quality decreasing the equilibrium cutoffs of other schools. It is (the sum over all other schools $c^{\prime}$ of) the product of the change in cutoffs of the other school $\left(-\frac{d P_{c^{\prime}}^{*}}{d \delta_{c}}\right)$, times the quantity of students in the margin that change schools due to a small change in cutoffs, $M_{c c^{\prime}}$, times the difference in the average quality of these students and the quality of a marginal student $\bar{P}_{c^{\prime} c}-P_{c}^{*}$. The market power effect from school $c^{\prime}$ has the same sign as $d P_{c^{\prime}}^{*} / d \delta_{c}$. It reduces the incentives to invest in quality if increasing $\delta_{c}$ reduces the selectivity of school $c^{\prime}$. However, it can be positive in the counterintuitive case where improving the quality of school $c$ increases the selectivity of school $c^{\prime}$. The latter case is only possible if $C \geq 3 .{ }^{27}$ The intuition for the direction of the market power effect is that improvements in quality help if they induce competing schools to become more selective, but harm in the more intuitive case where improving quality makes other schools less selective, and therefore compete more aggressively for students.

Hatfield et al.'s (2011a) main result is that, in a large thick market, where each school comprises a negligible fraction of the market, the incentives to invest in quality are weakly positive. Within our framework this can be interpreted as saying that, in such markets, the market power term becomes small, and therefore $d Q_{c} / d \delta_{c} \geq 0$.

Note that the breakdown of incentives in Equation (5.1) gives conditions where schools have muted incentives to invest in quality improvements for lower ranked students. If $\delta_{c}$ is a dimension of quality such that $d Y_{c}(e) / d \delta_{c} \approx 0$ unless $e_{c} \leq P_{c}$ or $e_{c} \approx P_{c}$, then the direct effect

$$
\int_{\epsilon_{c}}\left[e_{c}-P_{c}\right] \cdot \frac{d \bar{Y}_{c}}{d \delta_{c}}(P(\delta), \delta, e) d G(e) \approx 0 .
$$

Consider the case where the effect of the quality of school $c$ on the cutoffs of other schools is either small, as in a large market, or has the intuitive sign $d P_{c^{\prime}}^{*} / d \delta_{c} \leq 0$. Then the small direct effect and weakly negative market power effect imply $d Q_{c} / d \delta_{c} \leq 0$. Therefore, by allowing schools to competes, school choice gives incentives to invest in improvements benefiting the best students, but not the marginal accepted students. An

${ }^{27}$ To see this, write the aggregate demand function conditional on $\delta$ as $D(P, \delta)$. Then $D\left(P^{*}(\delta), \delta\right)=S$. By the implicit function theorem, we have $\partial_{\delta} P^{*}=-\left(\partial_{P} D\right)^{-1} \cdot \partial_{\delta} D$. If $C=2$, solving this system implies $d P_{c^{\prime}}^{*} / d \delta_{c} \leq 0$ for $c \neq c^{\prime}$. With $C=3$, an example of $d P_{c^{\prime}}^{*} / d \delta_{c}>0$ for $c \neq c^{\prime}$ is given by

$$
\partial_{P} D=\left(\begin{array}{ccc}
-10 & 1 & 1 \\
4 & -10 & 1 \\
4 & 1 & -10
\end{array}\right), \partial_{\delta_{1}} D=(10,-9,-1) .
$$

In this example the effect of increasing the quality of college 1 on cutoffs is $\partial_{\delta_{1}} P^{*}=(.98,-.49, .24)$, so that the cutoff of college 3 goes up with an increase in $\delta_{1}$. The intuition is that an increase in quality of college 1 takes more students from college 2 than college 3 , and the decrease in the selectivity of college 2 induces college 3 to become more selective. 
example would be that a school has incentives to invest in a better calculus teacher, and assigning counselor time to advise students in applying to top schools; and at the same time small or negative incentives to improve the quality of classes for lower ranked students, or invest counselor time in helping students with low grades. The logic of this result is that, since the quantity of students $S_{c}$ that are matched to school $c$ is fixed, for every student of score $e_{c}$ that a school gains by improving quality it must shed a marginal student with score $P_{c}^{*}$. The direct effect can only be profitable if $e_{c}-P_{c}^{*}$ is appreciably greater than 0 . The argument is completed by the observation that the market power term is weakly negative if $d P_{c^{\prime}}^{*} / d \delta_{c} \leq 0$. Note that marginal students with scores $e_{c} \approx P_{c}$ are not necessarily "bad". For a top-ranked special high school, cutoffs $P_{c}$ should be high, in the sense that a student of type $e_{c}$ is very desirable. Yet, since changes in quality only shift the composition of an entering class, it is still the case that the incentives to invest in attracting such students is small. Another way to frame this discussion is that the only scenario where the incentives to invest in marginal students may be positive is when a school does have market power, in the sense that it can affect the cutoffs of other schools, and for at least one of these other schools $d P_{c^{\prime}}^{*} / d \delta_{c}>0$.

The model yields an additional distortion. Even though quality affects $u_{c}^{i}\left(\delta_{c}\right)$ for all students, schools are only concerned with the impact on students who are indifferent between different schools, as Equation (5.1) only depends on changes in $Y$ and $f_{\delta}$. This is the familiar Spence (1975) distortion of a quality setting monopolist. Its manifestation in our setting is that schools' investment decisions take into account marginal but not inframarginal students.

Finally, if we assume that schools are symmetrically differentiated, it is possible to gain further intuition on the market power effect. If the function $f_{\delta}(\theta)$ is symmetric over schools, and all $S_{c}=S_{c^{\prime}}, \delta_{c}=\delta_{c^{\prime}}$, then the market power term reduces to

$$
-\frac{1}{C-1} \cdot \frac{M_{c^{\prime} c}}{M_{c \emptyset}+C \cdot M_{c^{\prime} c}} \cdot\left[\bar{P}_{c^{\prime} c}-P_{c}^{*}\right] \cdot \partial_{\delta_{c}} D_{c}\left(P^{*} \mid \eta_{\delta}\right),
$$

where $M_{c \emptyset}=\int_{\left\{\theta: e_{c}^{\theta}=P_{c}, e_{c^{\prime}}^{\theta}<P_{c^{\prime}} \forall c^{\prime} \neq c\right\}} f_{\delta}(\theta) d \theta$ is the $C-1$ dimensional mass of agents who are marginally accepted to school $c$ and not accepted to any other schools.

In the symmetric case, the market power effect is negative, and proportional to the quality wedge $\bar{P}_{c^{\prime} c}-P_{c}^{*}$ times the amount of students that school $c$ attracts with improvements in quality, $\partial_{\delta_{c}} D_{c}\left(P^{*} \mid \eta_{\delta}\right)$. Ceteris paribus, the absolute value of the market power effect grows with $M_{c^{\prime} c}$, the mass of students on the margin between school $c^{\prime}$ and $c$. These are the students that school $c$ may lose to $c^{\prime}$ if $c^{\prime}$ competes more aggressively. The absolute value of the market power effect also decreases with the number of schools $C$, and holding fixed the other quantities it converges to 0 quadratically as the number of schools grows. The equation suggests conditions under which schools having incentives 
to deteriorate quality for marginal applicants is a concern. This is the case when a small number of schools compete fiercely for densely populated margins $\tilde{M}_{c c^{\prime}}$. An example would be a city with a small number of elite schools, that compete for the best students but are horizontally differentiated, so that many students are in the margins $\tilde{M}_{c c^{\prime}}$.

The analysis in this Section could be extended in a number of ways. If the model specified costs for schools to invest, it would be possible to derive first order conditions for equilibrium play of schools. By specifying social welfare, the equilibrium conditions could be compared with optimization by a social planner. As the goal of this Section is simply to illustrate the derivation of comparative statics in the continuum framework, in the interest of space we leave these extensions for future research, and discuss related applications in the conclusion.

5.2. Random Economies and Convergence Rates. This section extends the convergence results to randomly generated finite economies. The results also give bounds on the speed of convergence of the set of stable matchings. These results are useful for performing simulations with randomly generated economies. Moreover, many mechanisms used in practical market design explicitly incorporate randomly generated preferences, so that the results imply new characterizations of the asymptotics of such mechanisms, which we explore in the next section.

We begin this section bounding the difference between market clearing cutoffs in a continuum economy and in a finite approximation.

Proposition 2. Assume that the continuum economy $E=[\eta, S]$ admits a unique stable matching $\mu$, and $\sum_{c} S_{c}<1$. Let $P^{*}$ be the associated market clearing cutoff, and assume $D(\cdot \mid \eta)$ is $C^{1}$, and $\partial D\left(P^{*}\right)$ is invertible. Then there exists $\alpha \geq 0$ such that for any finite economy $F=\left[\eta^{F}, S^{F}\right]$.

$$
\begin{array}{r}
\sup \left\{\left|P^{F}-P^{*}\right|: P^{F} \text { is a market clearing cutoff of } F\right\} \\
\leq \alpha \cdot\left(\sup _{P \in[0,1]^{C}}\left|D(P \mid \eta)-D\left(P \mid \eta^{F}\right)\right|+\left|S-S^{F}\right|\right) .
\end{array}
$$

The Proposition shows that the distance between market clearing cutoffs of a continuum economy and a discrete approximation is of the same order of magnitude as the distance between the associated vectors of capacities, plus the difference between the demand functions. Therefore, if the distance between the empirical distribution of types $\eta^{F}$ and $\eta$ is small, and the distance between the supply vectors $S^{F}$ and $S$ is small, the continuum model is guaranteed to provide a good approximation for finite economies.

We now extend the convergence in Theorem 2 to economies where agents are randomly drawn, with types independently and identically distributed. The following Proposition implies not only convergence of the sets of stable matchings, but also a 
strong bound on the rate of convergence. For a real number $x$ we denote by $\{x\}$ the nearest integer to $x$.

Proposition 3. Assume that the continuum economy $E=[\eta, S]$ admits a unique stable matching $\mu$, and $\sum_{c} S_{c}<1$. Let $F^{k}=\left[\eta^{k}, S^{k}\right]$ be a randomly drawn finite economy, with $k$ students drawn independently according to $\eta$ and the vector of capacity per student $S^{k}$ defined as $S^{k} k=\{S k\}$. Let $\left\{\tilde{\mu}^{k}\right\}_{k \in \mathbb{N}}$ be a sequence of random variables, such that each $\tilde{\mu}^{k}$ is a stable matching of $F^{k}$. We have the following results.

(1) $F^{k}$ converges almost surely to $E$, and $\tilde{\mu}^{k}$ converges almost surely to $\mu$.

(2) Take $\epsilon>0$. Let $P^{*}$ be the unique market clearing cutoff of $E$. If $D(\cdot \mid \eta)$ is $C^{1}$, and $\partial D\left(P^{*}\right)$ is invertible, then there exist constants $\alpha, \beta \geq 0$ such that probability that $F^{k}$ has a market clearing cutoff $P^{k}$ with $\left|P^{*}-P^{k}\right| \geq \epsilon$ is bounded by

$$
\operatorname{Pr}\left\{F^{k} \text { has a market clearing cutoff } P^{k} \text { with }\left|P^{k}-P^{*}\right| \geq \epsilon\right\} \leq \alpha \cdot e^{-\beta k} \text {. }
$$

(3) Moreover, if $\eta$ can be represented by a continuous density, let the $G^{k}$ be fraction of students in economy $F^{k}$ that receives a match different from that in the limit economy, that is, $D^{\theta}\left(P^{k}\right) \neq D^{\theta}\left(P^{*}\right)$ for some market clearing cutoff $P^{k}$ of $F^{k}$. Then $G^{k}$ converges to 0 almost surely, and there exist $\alpha^{\prime}, \beta^{\prime} \geq 0$ such that the probability that $G^{k} \geq \epsilon$ is bounded by

$$
\operatorname{Pr}\left\{G^{k} \geq \epsilon\right\} \leq \alpha^{\prime} \cdot e^{-\beta^{\prime} k}
$$

The first part of the Proposition says that the stable matchings of the randomly drawn economies converge almost surely to stable matchings of the limit approximation. This justifies using the continuum model as an approximation of the discrete model in settings where preference are random.

The second part of the Proposition gives bounds on how fast convergence takes place. Given $\epsilon>0$, the Corollary guarantees that the probability that market clearing cutoffs in $F^{k}$ deviate from those in $E$ by more than $\epsilon$ converges to 0 exponentially. Moreover, it guarantees that the fraction of students that may receive different matches in the continuum and finite economy is lower than $\epsilon$ with probability converging to 1 exponentially.

Crucially, the proofs of the bounds above are constructive. Therefore, besides providing an exponential rate of convergence, the results may be used to produce specific constants for convergence, as a function of $\eta$ and $S$. The proof of Proposition 2 starts by observing that the excess demand function $z(\cdot \mid E)=D(\cdot \mid E)-S$ must be bounded away from 0 outside of a neighborhood of $P^{*}$. It then uses the approximation of $z$ by its derivative to bound the distance market clearing cutoffs of economy $F$ to $P^{*}$. The proof of Proposition 3 uses the Glivenko-Cantelli Theorem to show almost sure convergence of the empirical distributions of types $\eta^{F}$ to $\eta$. Theorem 2 then guarantees almost sure 
convergence of stable matchings $\tilde{\mu}^{k}$ to $\mu$. The exponential bounds follow from Proposition 3, and from the Vapnik and Chervonenkis (1971) bounds from computational learning theory, that guarantee fast convergence of $D\left(P \mid F^{k}\right)$ to $D(P \mid E)$, uniformly in $P$.

5.3. Comparative Statics in Large Finite Markets. One of the advantages of the continuum model is that comparative statics can be derived using standard techniques. However, the applicability of the model depends on the comparative statics results extending to actual finite markets. The following Proposition guarantees that this is the case.

Proposition 4. Consider two limit economies $E, E^{\prime}$, with unique market clearing cutoffs $P, P^{\prime}$. Let $\left\{F^{k}\right\}_{k \in \mathbb{N}},\left\{F^{\prime k}\right\}_{k \in \mathbb{N}}$ be sequences of finite economies with $F^{k} \rightarrow E, F^{\prime k} \rightarrow$ $E^{\prime}$. Then there exists $k_{0}$ such that for all $k \geq k_{0}$ and any pair of market clearing cutoffs $P^{k}$ of $F^{k}$ and $P^{\prime k}$ of $F^{\prime k}$, if $P_{c}>P_{c}^{\prime}$ then $P^{k}>P^{\prime k}$, and if $P_{c}<P_{c}^{\prime}$ then $P^{k}<P^{\prime k}$.

The Proposition concerns continuum economies $E, E^{\prime}$ with unique stable matchings. It shows that if the market clearing cutoffs are ordered in a particular way, then the sets of market clearing cutoffs of approximating discrete economies are strongly ordered in the same way. Therefore, even though discrete economies may have several stable matchings, setwise comparative statics must be the same as in the continuum model.

\subsection{Market Design Applications.}

5.4.1. The Random Serial Dictatorship Mechanism. The assignment problem consists of allocating indivisible objects to a set of agents. No transfers of a numeraire or any other commodity are possible. The most well-known solution to the assignment problem is the random serial dictatorship (RSD) mechanism. In the RSD mechanism, agents are first ordered randomly by a lottery. They then take turns picking their favorite object, out of the ones that are left. Recently, Che and Kojima (2010) have characterized the asymptotic limit of the RSD mechanism. In their model, the number of object types is fixed, and the number of agents and copies of each object grows. Their main result is that RSD is asymptotically equivalent to the probabilistic serial mechanism proposed by Bogomolnaia and Moulin (2001). This is a particular case of our results, as the serial dictatorship mechanism is equivalent to deferred acceptance when all colleges have the same ranking over students. This section formalizes this point.

In the assignment problem there are $C$ object types $c=1,2, \ldots, C$, plus a null object $C+1$, which corresponds to not being assigned an object. A particular instance of the assignment problem is given by $A P=(k, m, S)$, where $k$ is the number of agents, $m$ is a vector with $m_{\succ}$ representing the fraction of agents with preferences $\succ$ for each $\succ \in \mathcal{R}$, 
and $S$ a vector with $S_{c}$ being the number of copies of object $c$ available per capita. An allocation specifies for each agent $\theta$ a probability $x^{\theta}(c \mid A P)$ of receiving each object $c$.

We can describe RSD as a particular case of the deferred acceptance mechanism where all colleges have the same preferences. First, we give agents priorities based on a lottery $l$, generating a random finite college admissions problem $F(A P, l)$, where agents correspond to students, and colleges to objects. Formally, given assignment problem AP, randomly assign each agent $\theta$ a single lottery number $l^{\theta}$ uniformly in $[0,1]$, that gives her score in all colleges (that is, objects) of $e_{c}^{\theta}=l$. This induces a random discrete economy $F(A P, l)$ as in the previous section. That is, as $l$ is a random variable, $F(A P, l)$ is a random finite economy, and for particular draws of $l$ it is a finite economy. For almost every draw of $l$ the economy $F(A P, l)$ has strict preferences. Each agent's allocation under RSD is then equal to her expected allocation, over all values of $l$, in $F(A P, l)$.

Consider now a sequence of finite assignment problems $\left\{A P^{k}\right\}_{k \in \mathbb{N}}, A P^{k}=\left(k, m^{k}, S^{k}\right)_{k \in \mathbb{N}}$. Assume $\left(m^{k}, S^{k}\right)$ converges to some $(m, S)$ with $S>0, m>0$. Let each $l^{k}$ be a lottery consisting of $k$ draws, one for each agent, randomly distributed in $[0,1]^{k}$. For each $k$, the assignment problem and the lottery induce a random economy $F\left(A P^{k}, l^{k}\right)$.

Note that the finite economies $F\left(A P^{k}, l^{k}\right)$ converge almost surely to a continuum economy $E$ with a vector $S$ of quotas, a mass $m_{\succ}$ of agents with each preference list $\succ$, and scores $e^{\theta}$ uniformly distributed along the diagonal of $[0,1]^{C}$. This limit economy has a unique market clearing cutoff $P(m, S)$. By Proposition 3, cutoffs in large finite economies are converging almost surely to $P(m, S)$. We have the following characterization of the limit of the RSD mechanism.

Proposition 5. Under the RSD mechanism the probability that an agent with preferences $\succ$ will receive object $c$ converges to

$$
\int_{l \in[0,1]} \mathbf{1}_{\left(c=\underset{\succ}{\left.\arg \max \left\{c \in C \mid P_{c}(m, S) \leq l\right\}\right)}\right.} d l .
$$

That is, the cutoffs of the limit economy describe the limit allocation of the RSD mechanism. In the limit, agents are given a lottery number uniformly drawn between 0 and 1 , and receive their favorite object out of the ones with cutoffs below the lottery number. Inspection of the market clearing equations shows that cutoffs correspond to 1 minus the times where objects run out in the probabilistic serial mechanism. This yields the Che and Kojima (2010) result on the asymptotic equivalence of RSD and the probabilistic serial mechanism.

5.4.2. School Choice Mechanisms. The Che and Kojima (2010) result works exclusively for random serial dictatorship, a mechanism where students are treated symmetrically, 
and do not have priorities for different objects. However, in mechanisms used in practice for the assignment of students to schools, agents do have priorities. This section generalizes the results to this setting, yielding an asymptotic characterization of school choice mechanisms used in actual school choice clearinghouses.

The school choice problem consists of assigning seats in public schools to students, while observing priorities some students may have to certain schools. It differs from the assignment problem because schools give priorities to subsets of students. It differs from the classic college admissions problem in that often schools are indifferent between large sets of students (Abdulkadiroglu and Sönmez 2003). For example, a school may give priority to students living within its walking zone, but treat all students within a priority class equally. In Boston and NYC, the clearinghouses that assign seats in public schools to students were recently redesigned by academic economists (Abdulkadiroglu et al. 2005a,b). The chosen mechanism was deferred acceptance with single tie-breaking (DA-STB). DA-STB first orders all students using a single lottery, which is used to break indifferences in the schools' priorities, generating a college admissions problem with strict preferences. It then runs the student-proposing deferred acceptance algorithm, given those refined preferences (Abdulkadiroglu et al. 2009; Kesten and Ünver 2010).

We can use our framework to derive the asymptotics of the DA-STB mechanism. Fix a set of schools $C=1, \ldots, C, C+1$ (which correspond to the colleges in our framework). School $C+1$ is the null school that corresponds to being unmatched, and is the least preferred school of each student. Student types $\bar{\theta}=\left(\succ^{\bar{\theta}}, e^{\bar{\theta}}\right)$ are again given by a strict preference list $\succ^{\bar{\theta}}$ and a vector of scores $e^{\bar{\theta}}$. However, to incorporate the idea that schools only have very coarse priorities, corresponding to a small number of priority classes, we assume that all $e_{c}^{\bar{\theta}}$ are integers in $\{0,1,2, \ldots, \bar{e}\}$ for $\bar{e} \geq 0$. Therefore, the set of possible student types is finite. We denote by $\bar{\Theta}$ the set of possible types. A school choice problem $S C=(k, m, S)$ is given by a number of students $k$, and a measure $m_{\bar{\theta}}$ of students of each of the finite types $\bar{\theta} \in \bar{\Theta}$, and a vector of capacity per capita of each school $S$.

We can describe the DA-STB mechanism as first breaking indifferences through a lottery $l$, which generates a finite college admissions model $F(S C, l)$, and then giving each student the student-proposing deferred acceptance allocation. Assume each student $\theta$ receives a lottery number $l^{\theta}$ independently uniformly distributed in $[0,1]$. The student's refined score in each school is given by her priority, given by her type, plus lottery number, $e_{c}^{\theta}=e_{c}^{\bar{\theta}}+l .^{28}$ Therefore, the lottery yields a randomly generated finite economy $F(S C, l)$, as defined in Corollary 3. The DA-STB mechanism then assigns each student in $F$ to her match in the unique student-optimal stable matching. For

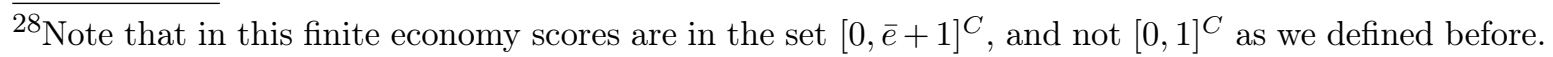
It is straightforward to extend the model to this setting.
} 
each type $\bar{\theta}$ in the original problem, denote by $x_{D A-S T B}(\bar{\theta} \mid S C)$ in $\Delta C$ the probability distribution over schools she receives over all realizations of $l$.

Consider now a sequence of school choice problems $S C^{k}=\left(k, m^{k}, S^{k}\right)$, each with $k \rightarrow \infty$ students. Problem $k$ has a fraction $m_{\bar{\theta}}^{k}$ of students of each type, and school $c$ has capacity $S_{c}^{k}$ per student. The null school has capacity $S_{C+1}=\infty$. Assume $\left(m^{k}, S^{k}\right)$ converges to some $(m, S)$ with $S>0, m>0$.

Analogously to the assignment problem, as the number of agents grows, the aggregate randomness generated by the lottery disappears. The randomly generated economies $F\left(S C^{k}, l^{k}\right)$ are converging almost surely to a limit economy, given as follows. For each of the possible types $\bar{\theta} \in \bar{\Theta}$, let the measure $\eta_{\bar{\theta}}$ over $\Theta$ be uniformly distributed in the line segment $\succ^{\bar{\theta}} \times\left[e^{\bar{\theta}}, e^{\bar{\theta}}+(1,1, \cdots, 1)\right]$, with total mass 1 . Let $\eta=\sum_{\bar{\theta} \in \bar{\Theta}} m_{\bar{\theta}} \cdot \eta_{\bar{\theta}}$. The limit continuum economy is given by $E=[\eta, S]$. We have the following generalization of the result in the previous section.

Proposition 6. Assume the limit economy $E$ has a unique market clearing cutoff $P(m, S)$. Then the probability that DA-STB assigns a student with type $\bar{\theta} \in \bar{\Theta}$ to school c converges to

$$
\int_{l \in[0,1]} \mathbf{1}_{\left(c=\underset{\succ}{\arg \max }\left\{c \in C \mid P_{c}(m, S) \leq e_{c}^{\theta}+l\right\}\right)} d l .
$$

The Proposition says that the asymptotic limit of the DA-STB allocation can be described using cutoffs. The intuition is that, after tie-breaks, a discrete economy with a large number of students is very similar to a continuum economy where students have lottery numbers uniformly distributed in $[0,1]$. The main limitation of the Proposition is that it requires the continuum economy to have a unique market clearing cutoff. Although we know that this is valid for generic vectors of capacities $S$, example 1 below shows that it is not always the case.

This result also suggests that the outcome of the DA-STB mechanism should display small aggregate randomness, even though the mechanism is based on a lottery. The Proposition suggests that, for almost every vector $(m, S)$, the market clearing cutoffs of large discrete economies approach the unique market clearing cutoff of the continuum limit. Therefore, although the allocation a student receives depends on her lottery number, she faces approximately the same cutoffs with very high probability. This is consistent with simulations using data from the New York City match, reported by Abdulkadiroglu et al. (2009). For example, they report that in multiple runs of the algorithm, the average number of applicants who are assigned their first choice is $32,105.3$, with a standard deviation of only 62.2 .

Another important feature of the Proposition is that the asymptotic limit of DA-STB given by cutoffs is analytically simpler than the allocation in a large discrete economy. To compute the allocation of DA-STB in a discrete economy, it is in principle necessary 
to compute the outcome for all possible ordering of the students by a lottery. Therefore, to compute the outcome with ten students, it is necessary to consider 10 ! $\approx 4 \cdot 10^{6}$ lottery outcomes, and for each one compute the outcome of the deferred acceptance algorithm. For an economy with 100 students, the number of possible lottery outcomes is $100 ! \approx 10^{156} \cdot{ }^{29}$ Consequently, the continuum model can be applied to derive analytic results on the outcomes of DA-STB in large economies. Azevedo and Leshno (2010) apply this model to compare the equilibrium properties of deferred acceptance with student-optimal stable mechanisms.

In addition, the Proposition generalizes the result in the previous section, that describes the asymptotic limit of the RSD mechanism. RSD corresponds to DA-STB in the case where all students have equal priorities. Therefore, the market clearing equations provide a unified way to understand asymptotics of RSD, the probabilistic serial mechanism, and DA-STB. Moreover, one could easily consider other ways in which the lottery $l$ is drawn, and derive asymptotics of other mechanisms, such as deferred acceptance with multiple tie-breaking discussed by Abdulkadiroglu et al. (2009).

5.5. Markets with Multiple Stable Matchings. Section 4 shows that generic continuum economies have a unique stable matching, and that there is a close connection between the stable matchings of the continuum and discrete model in that case. The reason why uniqueness is an important requirement is that, when the continuum economy admits more than one stable matching, these matchings may not be robust with respect to small perturbations in the economy. The following example illustrates this point.

\section{Example 1. (School Choice)}

This example is based on a school choice mechanism similar to DA-STB, described in Section 5.4. The preferences in the example are those that would arise in a school district where some students are given priorities, and these are broken with a single lottery. ${ }^{30}$

A city has two schools, $c=1,2$, with a quota of $S_{1}=S_{2}=1$. Students have priorities to schools according to the walk zones where they live in. A mass 1 of students lives in the walk zone of each school. In this example, the grass is always greener on the other side, so that students always prefer the school to which they don't have priority. To

\footnotetext{
${ }^{29}$ The fact that the distribution over outcomes induced by these mechanisms is much more complex in finite markets than in the continuum limit is also present in the particualr case of the assignment problem, where the allocations induced by random serial dictatorship are very complex (Manea (2009); Pycia and Liu (2011)).

${ }^{30}$ The example is a continuum version of an example used by Erdil and Ergin (2008) to show a shortcoming of deferred acceptance with single tie-breaking: it may produce matchings which are ex post inefficient with respect to the true preferences, before the tie-breaking. That is, the algorithm often produces allocations which are Pareto dominated by other stable allocations.
} 

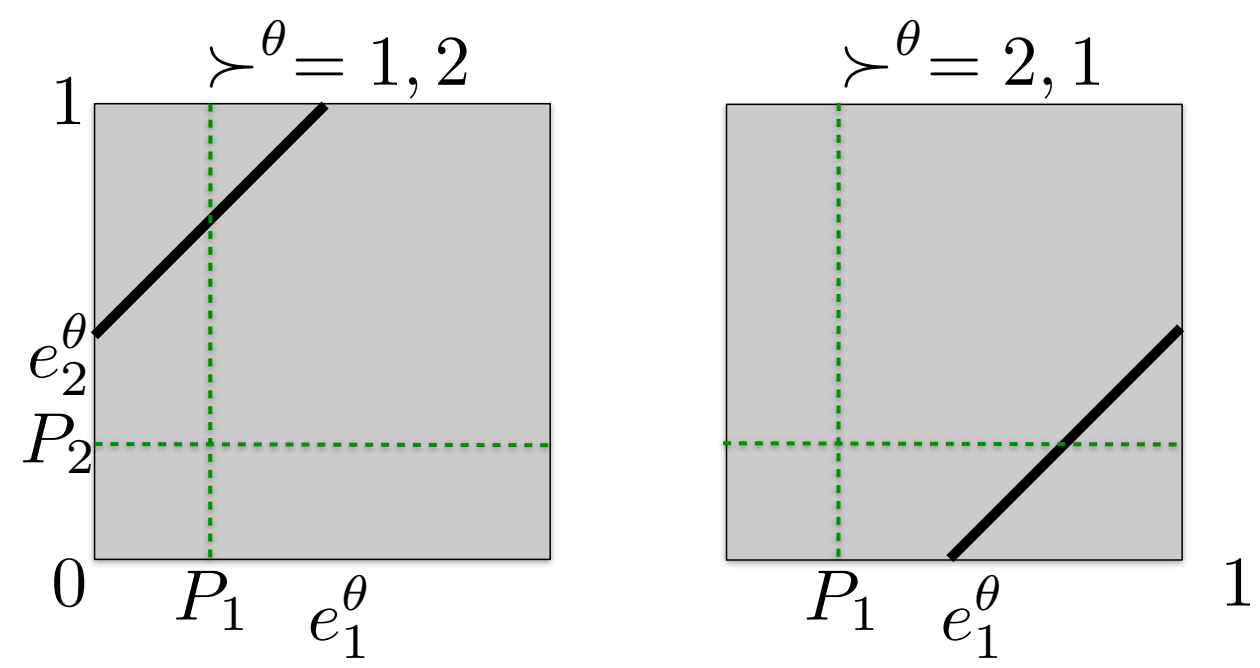

Figure 5. The distribution of student types in Example 1. The unit mass of students is uniformly distributed over the solid lines. The left square represents students in the walk zone of school 2, and the right square students with priority to school 1 . The dashed lines represents one of an infinite number of possible vectors of market clearing cutoffs $P_{1}=P_{2}$.

break ties, the city gives each student a single lottery number $l$ uniformly distributed in $[0,1]$. The student's score is

$$
l+I\left(\theta \text { is in } c^{\prime} \text { s walk zone }\right) .
$$

Figure 5 depicts the distribution of students in the economy.

Note that market clearing cutoffs must be in $[0,1]$, as the mass of students with priority to each school is only large enough to exactly fill each school. Consequently, the market clearing equations can be written

$$
\begin{aligned}
& 1=S_{1}=\left(1-P_{1}\right)+P_{2} \\
& 1=S_{2}=\left(1-P_{2}\right)+P_{1} .
\end{aligned}
$$

The first equation describes demand for school 1. $1-S_{1}$ students in the walk zone of 2 are able to afford it, and that is the first term. Also, $P_{2}$ students in the walk zone of 1 would rather go to 2, but don't have high enough lottery number, so they have to stay in school 1. The market clearing equation for school 2 is the same.

These equations are equivalent to

$$
P_{1}=P_{2} .
$$

Hence any point in the line $\{P=(x, x) \mid x \in[0,1]\}$ is a market clearing cutoff - the lattice of stable matchings has infinite points, ranging from a student-optimal stable matching, $P=(0,0)$ to a school-optimal stable matching $P=(1,1)$. 
Now modify the economy by adding a small mass of agents that have no priority, so that the new mass has $e^{\theta}$ uniformly distributed in $[(0,0),(1,1)]$. It's easy to see that in that case the unique stable matching is $P=(1,1)$. Therefore, adding this small mass undoes all stable matchings except for $P=(1,1)$. In addition, it is also possible to find perturbations that undo the school-optimal stable matching $P=(1,1)$. If we add a small amount $\epsilon$ of capacity to school 1 , the unique stable matching is $P=(0,0)$. And if we reduce the capacity of school 1 by $\epsilon$, the unique stable matching is $P=(1+\epsilon, 1)$, which is close to $P=(1,1)$.

The following Proposition generalizes the example. It shows that, when the set of stable matchings is large, then none of the stable matchings are robust to small perturbations. The statement uses the fact, proven in Appendix A, that for any economy $E$ there exists a smallest and a largest market clearing cutoff, in the sense of the usual partial ordering of $\mathbb{R}^{C}$.

Proposition 7. (Non Robustness) Consider an economy $E$ with more than one stable matching and $\sum_{c} S_{c}<1$. Let $P$ be one of its market clearing cutoffs. Assume $P$ is either strictly larger than the smallest market clearing cutoff $P^{-}$, or strictly smaller than the largest $P^{+}$. Let $N$ be a sufficiently small neighborhood of $P$. Then there exists a sequence of economies $E^{k}$ converging to $E$ without any market clearing cutoffs in $N$.

Proof. Suppose $P>P^{-}$; the case $P<P^{+}$is analogous. Assume $N$ is small enough such that all points $P^{\prime} \in N$ satisfy $P^{\prime}>P^{-}$. Denote $E=[\eta, S]$, and let $E^{k}=\left[\eta, S^{k}\right]$, where $S_{c}^{k}=S_{c}+1 / C k$. Consider a sequence $\left\{P^{k}\right\}_{k \in \mathbb{N}}$ of market clearing cutoffs of $E^{k}$. Then

$$
\sum_{c \in C} D_{c}\left(P^{k} \mid \eta\right)=\frac{1}{k}+\sum S_{c} .
$$

However, for all points $P^{\prime}$ in $N$,

$$
\sum_{c \in C} D_{c}\left(P^{\prime} \mid \eta\right) \leq \sum_{c \in C} D_{c}\left(P^{-} \mid \eta\right)=\sum S_{c}<\sum S_{c}^{k} .
$$

However, for large enough $k, \sum S_{c}^{k}<1$, which means that for any market clearing cutoff $P^{k}$ of $E^{k}$ we must have $D\left(P^{k} \mid \eta\right)=S_{c}^{k}$, and therefore there are no market clearing cutoffs in $N$.

5.6. Transferable Utility and Matching with Contracts. In many markets, agents must negotiate not only who matches with whom, but also wages and other terms of contracts. When hiring faculty most universities negotiate both in wages and teaching load. Firms that supply or demand a given production input may negotiate, besides the price, terms like quality or timeliness of the deliveries. This section extends the continuum model to include these possibilities. Remarkably, it is still the case that stable matchings have the simple cutoff structure as described above. The extension 
permits the comparison of outcomes under different market institutions, of personalized versus uniform wages, a topic that is pursued in detail in Azevedo (2011).

5.6.1. The Setting. Formally, we now consider a set of doctors $\Theta$ distributed according to a measure $\eta$, a finite set of hospitals $H$, with $H$ also denoting the number of hospitals, and a set of contracts $X . \eta$ is assumed to be defined over a $\sigma$-algebra $\Sigma^{\Theta}$. Each contract $x$ in $X$ specifies

$$
x=(\theta, h, w),
$$

that is, a doctor, a hospital, and other terms of the contract $w$. A case of particular interest, to which we return to later, is when $w$ is a wage, and agents have quasilinear preferences.

We assume that $X$ contains a null contract $\emptyset$, that corresponds to being unmatched. A matching is a function

$$
\mu: \Theta \cup H \rightarrow X \cup 2^{X}
$$

that associates each doctor (hospital) to a (set of) contract(s) that contain it, or to the empty contract. In addition, each doctor can be assigned to at most one hospital. Moreover, hospitals must be matched to a set of doctors of measure at most equal to its capacity $S_{h}$. Finally, a matching has to be measurable with respect to the product $\sigma$-algebra given by $\Sigma^{\Theta}$ in the set $\Theta$ and the $\sigma$-algebra $2^{H}$ in the set of hospitals.

Models of matching with contracts have been proposed by Kelso and Crawford (1982); Hatfield and Milgrom (2005). ${ }^{31}$ Those papers define stable matchings with respect to preferences of firms over sets of contracts. We focus on a simpler model, where stability is defined with respect to preferences of firms over single contracts. This corresponds to the approach that focuses on responsive preferences in the college admissions problem. This restriction considerably simplifies the exposition, as the same arguments used in the previous sections may be applied. Henceforth we assume that hospitals have preferences over single contracts that contain it, and the empty contract $\succ^{h}$, and agents have preferences over contracts that contain them and over being unmatched $\succ^{\theta}$.

A single agent (doctor or hospital) blocks a matching $\mu$ if it is matched to a contract that is worse than the empty contract. A matching is individually rational if no single agent blocks it. A doctor-hospital pair $\theta, h$ is said to block a matching $\mu$ if they are not matched, and there is a contract $x=(\theta, h, w)$ that $\theta$ prefers over $\mu(\theta)$ and either (i) hospital $h$ did not fill its capacity $\eta(\mu(h))<S_{h}$ and $h$ prefers $x$ to the empty contract, or (ii) $h$ is matched to a contract $x^{\prime} \in \mu(h)$ which it likes strictly less than contract $x$.

\footnotetext{
$\overline{{ }^{31} \text { See Sönmez }}$ and Switzer (2011); Sönmez (2011) for applications of these models to real-life market design problems.
} 
Definition 4. A matching $\mu$ is stable if it is individually rational and has no blocking pairs.

Assume that doctors' preferences can be expressed by a utility function $u^{\theta}(x)$, and hospitals' by a utility function $\pi_{h}(x)$. The utility of being unmatched is normalized to 0 . To get an analogue of the Supply and Demand Lemma, we impose some additional restrictions. Let $X_{h}^{\theta}$ be the set of contracts that contain both a hospital $h$ and a doctor $\theta$. A doctor (hospital) is said to be unacceptable to a hospital (doctor) if for all $x \in X_{h}^{\theta}$ we have $\pi_{h}(x)<0\left(u^{\theta}(x)<0\right)$. Let $M$ be a positive real number.

\section{Assumption 2. (Regularity Conditions)}

- (Boundedness) For any doctor-hospital pair $\theta, h$, and $x \in X_{\mu}^{\theta}: u^{\theta}(x)$ and $\pi_{h}(x)$ are contained in $[-M, M]$.

- (Compactness) For any doctor-hospital pair $\theta, h$ the set of pairs

$$
\left\{\left(u^{\theta}(x), \pi_{h}(x)\right) \mid x \in X_{h}^{\theta}\right\}
$$

is compact.

- (No Redundancy) Given $\theta, h$, no contract in $x \in X_{h}^{\theta}$ weakly Pareto dominates, nor has the same payoffs as another contract $x^{\prime} \in X_{h}^{\theta}$.

- (Completeness) Given $h$ and $k \in[-M, M]$, there exists an agent $\theta \in \Theta$ whose only acceptable hospital is $h$, and $\sup _{x: u^{\theta}(x) \geq 0} \pi_{h}(x)=\sup _{x: u^{\theta}(x)>0} \pi_{h}(x)=k$.

- (Measurability) Given any Lebesgue measurable set $K$ in $\mathbb{R}^{2}$ and $h \in H$, the $\sigma$-algebra $\Sigma^{\Theta}$ contains all points and all sets of the form

$$
\left\{\theta \in \Theta \mid K=\left\{\left(u^{\theta}(x), \pi_{h}(x)\right) \mid x \in X_{h}^{\theta}\right\}\right\} .
$$

5.6.2. Cutoffs. Within our matching with contracts framework, the allocation of doctors to hospitals is determined by an $H$-dimensional vector of cutoffs. It is convenient to think of cutoffs as the marginal value of capacity at each hospital - how much utility the hospital would gain from a small increase in capacity. Cutoffs are just numbers $P_{h} \in[0, M]$, and a vector of cutoffs $P \in[0, M]^{H}$.

We denote an agent's maximum utility of working for a hospital $h$ and providing the hospital with utility of at least a cutoff $P_{h}$ as

$$
\begin{aligned}
\bar{u}_{h}^{\theta}(P)= & \sup u^{\theta}(x) \\
\text { s.t. } & x \in X_{h}^{\theta} \\
& \pi_{h}(x) \geq P_{h} .
\end{aligned}
$$

We refer to this as the reservation utility that hospital $h$ offers doctor $\theta$. Note that the reservation utility may be $-\infty$ if the feasible set $\left\{x \in X_{h}^{\theta}: \pi_{x}(x) \geq P_{h}\right\}$ is 
empty. Moreover, whenever this sup is finite, it is attained by some contract $x$, due to the compactness assumption. We will define $\bar{u}_{\emptyset}^{\theta}(\cdot) \equiv 0$.

Now we define a doctor's demand. Note that doctors demand hospitals, and not contracts. The demand of a doctor $\theta$ given a vector of cutoffs $P$ is

$$
D^{\theta}(P)=\arg \max _{H \cup\{\emptyset\}} \bar{u}_{h}^{\theta}(P),
$$

Demand may not be uniquely defined, as an agent may have the same reservation utility in more than one hospital. Henceforth we assume that, given cutoffs, indifferences only occur for a measure 0 set of doctors.

Assumption 3. (Strict Preferences) For any cutoff vector $P \in \mathbb{R}$, and hospitals $h, h^{\prime}$, the set of agents with $\bar{u}_{h}^{\theta}(P)=\bar{u}_{h^{\prime}}^{\theta}(P) \neq-\infty$ has measure 0 .

From now on, we fix a selection from the demand correspondence, so that it is a function. The aggregate demand for a hospital is defined as

$$
D_{h}(P)=\eta\left(\left\{D^{\theta}(P)=h\right\}\right) .
$$

Note that $D_{h}(P)$ does not depend on the demand of agents which are indifferent between more than one hospital, by the strict preferences assumption.

A market clearing cutoff is defined exactly as in definition 2. Given a stable matching $\mu$, let $P=\mathcal{P} \mu$ be given by

$$
P_{h}=\inf \left\{\pi_{h}(x) \mid x \in \mu(h)\right\},
$$

if $\eta(\mu(h))=S_{h}$ and $P_{h}=0$ otherwise. Given a market clearing cutoff $P$, we define $\mu=\mathcal{M} P$ as follows. Consider first a doctor $\theta$. If $D^{\theta}(P)=\emptyset$, then $\theta$ is unmatched: $\mu(\theta)=\emptyset$. If $D^{\theta}(P)=h \in H$, then $\mu(\theta)$ is defined as the contract that gives the highest payoff to $h$ conditional on $\theta$ not having a better offer elsewhere. Formally,

$$
\begin{aligned}
\mu(\theta) & =\arg \max _{x \in X_{h}^{\theta}} \pi_{h}(x) \\
\text { s.t. } & u^{\theta}(x) \geq \bar{u}_{h^{\prime}}^{\theta}(P) \text { for all } h^{\prime} \neq h,
\end{aligned}
$$

Note that $\mu(\theta)$ is uniquely defined, by the compactness and no redundancy assumptions. Since we defined $\mu(\theta)$ for all doctors, we can uniquely define it for each hospital as

$$
\mu(h)=\{\theta: \mu(\theta)=(\theta, h, w) \text { for some } w\} .
$$

We have the following extension of the Supply and Demand Lemma.

Lemma 3. (Supply and Demand Lemma with Contracts) If $\mu$ is a stable matching, then $\mathcal{P} \mu$ is a market clearing cutoff, and if $P$ is a market clearing cutoff then $\mathcal{M P}$ is a stable matching. Moreover, $\mathcal{P} \mathcal{M}$ is the identity. 
Note that, in the matching with contracts setting, there is no longer a bijection between market clearing cutoffs and stable matchings. This happens because the contract terms $w$ are not uniquely defined, and because we have not imposed continuity conditions ruling out measure 0 multiplicities for doctors that are indifferent between two hospitals given cutoffs.

5.6.3. Existence. To establish the existence of a stable matching, we must modify the previous argument, which used the deferred acceptance algorithm. One simple modification is using a version of the algorithm that Biró (2007) terms a "score limit algorithm", which calculates a stable matching by progressively increasing cutoffs to clear the market. A straightforward application of Tarski's fixed point Theorem gives us existence in this case.

Proposition 8. A stable matching with contracts always exists.

Proof. Consider the operator $T:[0, M]^{H} \rightarrow[0, M]^{H}$ defined by $P^{\prime}=T P$ is the smallest solution $P^{\prime} \in[0, M]^{H}$ to the system of inequalities

$$
D_{h}\left(P_{h}^{\prime}, P_{-h}\right) \leq S_{h} .
$$

$T$ is weakly increasing in $P$. Moreover, it takes the cube $[0, M]^{H}$ in itself. By Tarski's fixed point Theorem, it has a fixed point, which must be a market clearing cutoff.

5.6.4. The Quasilinear Case. A particularly interesting case of the model is when contracts only specify a wage $w$, and preferences are quasilinear. That is, the utility of a contract $x=(\theta, h, w)$ is just

$$
\begin{aligned}
& u^{\theta}(x)=u_{h}^{\theta}+w \\
& \pi_{h}(x)=\pi_{h}^{\theta}-w .
\end{aligned}
$$

and contracts include all possible $w$ s, such that these values are in $[-M, M]$. Define the surplus of a doctor-hospital pair as

$$
s_{h}^{\theta}=u_{h}^{\theta}+\pi_{h}^{\theta} .
$$

If we assume that $M$ is large enough so that, for all $\theta$ in the support of $\eta$ we have $0 \leq s_{i}^{\theta} \leq M$, then doctors and hospitals may freely divide the surplus of a relationship. We will denote a model satisfying the above properties by a matching with contracts model with quasilinear preferences. From the definition of reservation utility we get that for all doctors in the support of $\eta$

$$
\bar{u}_{h}^{\theta}(p)=s_{h}^{\theta}-P_{h} .
$$

Therefore, in any stable matching, doctors are sorted into the hospitals where $s_{h}^{\theta}-P_{h}$ is the highest, subject to it being positive. One immediate consequence is that doctors 


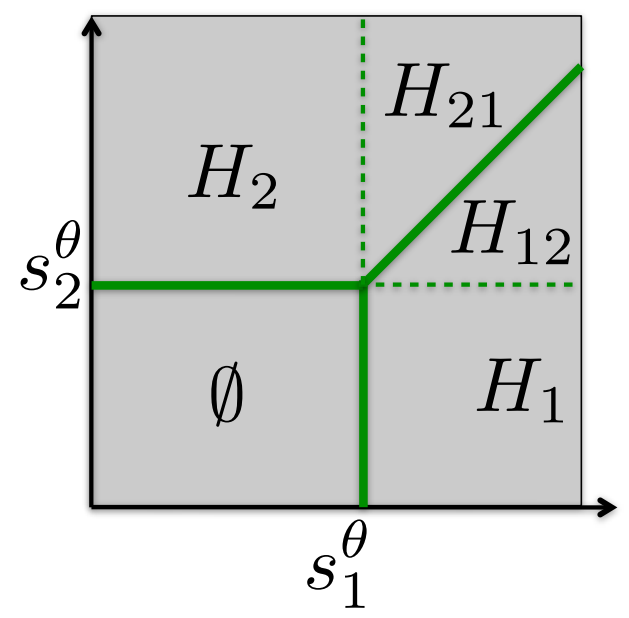

Figure 6. A matching with transferable utility with two hospitals. The square represents the set of possible surplus vectors $s^{\theta}$. Doctors in regions $H_{1}$ and $H_{12}$ are matched to hospital 1 , and doctors in regions $H_{2}$ and $H_{21}$ to hospital 2.

do not go necessarily to the hospital where they generate the largest surplus $s_{h}^{\theta}$. If $P_{h} \neq P_{h^{\prime}}$, it may be the case that $s_{h}^{\theta}>s_{h^{\prime}}^{\theta}$, but doctor $\theta$ is assigned to $h^{\prime}$. However, the allocation of doctors to hospitals does maximize the total surplus generated in the economy, in a sense made precise in Azevedo (2011) Appendix A.2. Figure 6 plots a stable matching in an economy with two hospitals.

Let the distribution of surplus vectors $s^{\theta}$ be $\eta_{s}$. We then have the following uniqueness result.

Proposition 9. Consider a matching with contracts model with quasilinear preferences. If $\eta_{S}$ has full support over $[0, M]$ then there is a unique vector of market clearing cutoffs.

The Proposition guarantees that the allocation of doctors to hospital is unique, up to a measure 0 set of doctors. However, the stable matching is not unique, as wages are not uniquely determined by stability. The intuition is that in a stable matching a hospital may offer a doctor any wage, as long as the doctor's utility is above that in her next best choice, and the hospital's gain from the relationship above its reservation value of capacity.

\section{Conclusion}

This paper proposes a new model of matching markets with a large number of agents on one side. The model admits complex heterogeneous preferences, as in the Gale and Shapley (1962) framework. However, it allows for straightforward derivation of comparative statics, as stable matchings are the solution to a set of supply and demand equations. We show that the model corresponds to the limit of large finite markets, 
that stable matchings are essentially unique in such economies, and apply the model to derive asymptotics of mechanisms used in practical market design, and to understanding incentives for schools to invest in quality when they compete for students. In these closing remarks, we highlight three important points that were not addressed in the analysis.

First, in recent years the matching literature has exploited more general frameworks than the Gale and Shapley model, on which we focus. These include non-responsive preferences, many-to-many matching, markets with more than two sides, and externalities between agents. ${ }^{32}$ In these models, the very existence of stable matchings depends on complex restrictions on preferences, such as substitutability. ${ }^{33}$ It would be interesting to understand to what extent the continuum of traders assumption obviates the need for such restrictions. ${ }^{34,35}$ This is specially important given Aumann's (1964) argument that price taking is only justified in a continuum setting, where agents indeed cannot affect prices. Analogously, stability is an inherently inconsistent solution concept in small finite markets. ${ }^{36}$

Second, a number of recent papers have empirically estimated matching models with heterogeneous preferences, using different methodologies. Bajari and Fox (2005) use the inequalities from the stability condition to gauge the efficiency of FCC auctions. Ho (2009) considers a strategic bargaining game as opposed to a frictionless stability notion to study insurer-hospital networks. In an analysis of venture capital firms Sorensen (2007) imposes restrictions on preferences so that the Gale and Shapley model has a unique stable matching, and his structural model has a well defined likelihood function which he uses for estimation. A natural extension of our model is to specify an empirical model for preferences. Since stable matchings are unique in our framework, such a model implies given parameters a distribution of colleges to which an agent is matched

\footnotetext{
${ }^{32}$ See respectively Kelso and Crawford (1982); Hatfield and Milgrom (2005) Echenique and Oviedo (2006); Roth (1984), Ostrovsky (2008); Hatfield et al. (2011b), and Sasaki and Toda (1996); Epple and Romano (1998).

${ }^{33}$ See Hatfield and Milgrom (2005); Hatfield and Kojima (2010) and Hatfield et al. (2011b).

${ }^{34}$ Starr (1969) demonstrates the existence of approximate competitive equilibria in large markets without convex preferences. See also Azevedo et al. (2011), who show that, in general equilibrium with indivisible commodities and quasilinear preferences, the continuum of traders assumption guarantees existence without the usual assumption of gross substitutes preferences made in the literature (Gul and Stacchetti 1999).

${ }^{35}$ See Ashlagi et al. (2011) and Kojima et al. (2010) for existence with stable matchings with complementarities in large markets.

${ }^{36}$ It is not the case, in general, that agents should report their preferences truthfully to a mechanism that implements a stable matching. Roth (1982) shows that there is no strategyproof and stable mechanism in the marriage problem. In large thick markets, Immorlica and Mahdian (2005); Kojima and Pathak (2009); Lee (2011) show that stable mechanisms are approximately strategyproof, in a sense precisely defined therein. Ehlers (2007) studies a far-sighted notion of stability, which may be more compelling in small markets.
} 
conditional on her observable characteristics. ${ }^{37}$ Such an approach requires assuming that the market is large enough to be well approximated by our continuum model. It would be interesting in further work to examine conditions under which such models are credibly identified and estimated. Moreover, it would be important to understand the advantages and disadvantages of using such a model for empirical work, versus the alternatives in the literature.

Third, a large share of the industrial organization literature is concerned with the strategic behavior of firms. Yet, through most of our analysis the characteristics of each college are held fixed. Even in our analysis of the incentives to invest in school quality we examined the effect of changes in quality, but did not consider the determination of Nash equilibria in a game where schools choose quality. One of the advantages of the continuum model is that it permits tractable analysis of this type of game. Azevedo (2011) and Veiga and Weyl (2011) apply our model to study the equilibrium behavior of firms, in two-stage games where firms first make strategic choices such as capacities or the degree of specialization, and in the second stage a matching is determined by stability. Such two-stage games are in the same spirit of the Cournot model, where in the first stage firms strategically commit to capacities, and in the second stage prices are given by market clearing. The industrial organization literature has several other models in this fashion, where firms first make a decision - such as quantity, capacity investments, quality, or product differentiation - and then prices and allocations are given by market clearing. Examples include specifications of Hotteling competition, and Klemperer and Meyer's (1989) supply function equilibrium. In matching markets, the concept of stability plays a role similar to market clearing. Since strategic behavior in these markets is still considerably less understood, a promising avenue is to consider such two-stage specifications, where firms make strategic choices and the allocation is given by stability.

The common theme in our analysis is applying market clearing conditions, as is standard in competitive equilibrium analysis, to matchings markets. This core idea permeates our characterization of stable matchings in terms of supply and demand equations, the decomposition of the effect of improving school quality in a direct and a market power effect, and characterizing the asymptotics of school choice mechanisms in terms of cutoffs. We hope this underlying idea will prove useful in the analysis of other market design problems. Furthermore, that it will broaden the applicability of Gale and

\footnotetext{
${ }^{37}$ For example, let $Z_{i c}$ be a vector of student $i$ and college $c$ observables. A simple model has a continuum mass of students with some distribution over observables, and preferences given by $u_{c}^{i}=$ $f\left(Z_{i c}, \epsilon_{i}, \xi_{c}, \alpha\right)$, and colleges have preferences given by $\pi_{c}^{i}=g\left(Z_{i c}, \epsilon_{i}, \xi_{c}, \beta\right)$, where $\epsilon_{i}$ and $\xi_{c}$ are random variables representing unobserved characteristics, and $\alpha$ and $\beta$ parameters to be estimated. If it is assumed for example that $u_{c}^{i}$ and $\pi_{c}^{i}$ have a non-atomic distribution with full support in $[0,1]^{2 C}$ for any fixed $\xi, \alpha \beta$, then our model has a unique stable matching, and for each vector of observables $Z_{i c}$ there is a well-defined probability of being matched to each college $c$.
} 
Shapley's (1962) notion of stability, yielding insights in specific markets where Becker's (1973) assumptions of vertical preferences and assortative matching do not hold. 


\section{REFERENCES}

Abdulkadiroglu, A. and T. Sönmez, "School Choice: A Mechanism Design Approach," American economic review, 2003, 93 (3), 729.

, P.A. Pathak, A.E. Roth, and T. Sönmez, "The Boston Public School Match," American Economic Review, 2005, 95 (2).

_, , and _ _ "The New York City High School Match," American Economic Review, 2005, 95 (2), 364-367.

, and _ "Strategy-Proofness Versus Efficiency in Matching with Indifferences: Redesigning the Nyc High School Match," American Economic Review, 2009, 99 (5), 1954-1978.

, Y.K. Che, and Y. Yasuda, "Expanding "Choice" in School Choice," 2008. http://papers.ssrn.com/sol3/papers. cfm?abstract_id=1308730.

Adachi, H., "On a Characterization of Stable Matchings," Economics Letters, 2000, 68 (1), 43-49.

Ashlagi, I., M. Braverman, and A. Hassidim, "Matching with Couples Revisited," in "Proceedings of the 12th ACM conference on Electronic commerce" ACM 2011, pp. 335-336.

Asker, J. and A. Ljungqvist, "Competition and the Structure of Vertical Relationships in Capital Markets," Journal of Political Economy, 2010, 118 (3), 599-647.

Aumann, R.J., "Markets with a Continuum of Traders," Econometrica, 1964, pp. 3950 .

Azevedo, E.M., "Imperfect Competition in Two-Sided Matching Markets," 2011. Mimeo, Harvard University.

and E.B. Budish, "Strategyproofness in the Large as a Desideratum for Market Design," 2011. Mimeo, University of Chicago Booth Graduate School of Business.

and J. Leshno, "Can We Make School Choice More Efficient? An Incentives Approach," 2010. Mimeo, Harvard University.

, E.G. Weyl, and A. White, "General Equilibrium without Gross Substitutes," 2011. http://papers.ssrn.com/so13/papers.cfm?abstract_id=1831944.

Bajari, P. and J.T. Fox, "Measuring the Efficiency of an FCC Spectrum Auction," 2005. NBER Working Paper No. 11671.

Balinski, M. and T. Sönmez, "A Tale of Two Mechanisms: Student Placement," Journal of Economic Theory, 1999, 84 (1), 73-94.

Becker, G.S., "A Theory of Marriage: Part I," Journal of Political Economy, 1973, pp. 813-846.

, "A Theory of Marriage: Part II," V Journal of Political Economy, 1974, 82 (2), S11-26. 
Biró, P., "Higher Education Admission in Hungary by a "Score-Limit Algorithm"," in "in" 18th International Conference on Game Theory at Stony Brook University 2007.

Bodoh-Creed, A., "Approximation of Large Games," 2010. Mimeo, Cornell University.

Bogomolnaia, A. and H. Moulin, "A New Solution to the Random Assignment Problem," Journal of Economic Theory, 2001, 100 (2), 295-328.

Budish, E.B., "The Combinatorial Assignment Problem: Approximate Competitive Equilibrium from Equal Incomes," Journal of Political Economy, Forthcoming.

and E. Cantillon, "The Multi-Unit Assignment Problem: Theory and Evidence from Course Allocation at Harvard," American Economic Review, Forthcoming.

Che, Y. and F. Kojima, "Asymptotic Equivalence of Probabilistic Serial and Random Priority Mechanisms," Econometrica, 2010.

Chiappori, P.A., M. Iyigun, and Y. Weiss, "Investment in Schooling and the Marriage Market," American Economic Review, 2009, 99 (5), 1689-1713.

Clark, S., "The Uniqueness of Stable Matchings," BE Journal of Theoretical Economics, 2006, 6 (1), 8 .

Coles, P., A. Kushnir, and M. Niederle, "Preference Signaling in Matching Markets," 2011. NBER Working Paper No. 16185.

, J. Cawley, P.B. Levine, M. Niederle, A.E. Roth, and J.J. Siegfried,

"The Job Market for New Economists: A Market Design Perspective," Journal of Economic Perspectives, 2010, 24 (4), 187-206.

Crawford, V.P., "Comparative Statics in Matching Markets," Journal of Economic Theory, 1991, 54 (2), 389-400.

Debreu, G., "Economies with a Finite Set of Equilibria," Econometrica, 1970, pp. 387392.

Devroye, L., L. Györfi, and G. Lugosi, A Probabilistic Theory of Pattern Recognition, Springer Verlag, 1996.

Dvoretzky, A., J. Kiefer, and J. Wolfowitz, "Asymptotic Minimax Character of the Sample Distribution Function and of the Classical Multinomial Estimator," Annals of Mathematical Statistics, 1956, pp. 642-669.

Echenique, F., "Contracts Vs. Salaries in Matching," American Economic Review, Forthcoming.

and J. Oviedo, "Core Many-to-One Matchings by Fixed-Point Methods," Journal of Economic Theory, 2004, 115 (2), 358-376.

and __ "A Theory of Stability in Many-to-Many Matching Markets," Theoretical Economics, 2006.

Eeckhout, J., "On the Uniqueness of Stable Marriage Matchings," Economics Letters, 
2000, 69 (1), 1-8.

Ehlers, L., "Von Neumann-Morgenstern Stable Sets in Matching Problems," Journal of Economic Theory, 2007, 134 (1), 537-547.

Epple, D. and R.E. Romano, "Competition between Private and Public Schools, Vouchers, and Peer-Group Effects," American Economic Review, 1998, pp. 33-62.

Erdil, A. and H. Ergin, "What's the Matter with Tie-Breaking? Improving Efficiency in School Choice," American Economic Review, 2008, 98 (3), 669-689.

Gabaix, X. and A. Landier, "Why Has Ceo Pay Increased so Much?," Quarterly Journal of Economics, 2008, 123 (1), 49.

Gale, D. and LS Shapley, "College Admissions and the Stability of Marriage," American Mathematical Monthly, 1962, 69 (1), 9-15.

Ginsburg, T. and J.A. Wolf, "The Market for Elite Law Firm Associates," Florida State University Law Review, 2003, 31, 909.

Grossman, G. and G. Maggi, "Trade and Diversity," American Economic Review, 2000, 90 (5).

Grossman, G.M., "The Distribution of Talent and the Pattern and Consequences of International Trade," Journal of Political Economy, 2004, pp. 209-239.

Guillemin, V. and A. Pollack, Differential Topology, Prentice-Hall Englewood Cliffs, NJ, 1974.

Gul, F. and E. Stacchetti, "Walrasian Equilibrium with Gross Substitutes," Journal of Economic Theory, 1999, 87 (1), 95-124.

Hatfield, J.W. and F. Kojima, "Substitutes and Stability for Matching with Contracts," Journal of Economic Theory, 2010.

and P.R. Milgrom, "Matching with Contracts," American Economic Review, 2005, 95 (4), 913-935.

, F. Kojima, and Y. Narita, "Promoting School Competition through School Choice: A Market Design Approach," 2011. Mimeo, Stanford Graduate School of Business.

, S.D. Kominers, A. Nichifor, M. Ostrovsky, and A. Westkamp, "Stability and Competitive Equilibrium in Trading Networks," 2011. Mimeo, Stanford Graduate School of Business.

Hitsch, G., A. Hortacsu, and D. Ariely, "Matching and Sorting in Online Dating," American Economic Review, 2010.

Ho, K., "Insurer-Provider Networks in the Medical Care Market," American Economic Review, 2009, 99 (1), 393-430.

Hoxby, C.M., "Does Competition among Public Schools Benefit Students and Taxpayers?," American Economic Review, 2000.

, "School Choice and School Competition: Evidence from the United States," 
Swedish Economic Policy Review, 2003, 10 (2), 9-66.

Hurwicz, L., "On Informationally Decentralized Systems," Decision and organization: A volume in honor of Jacob Marschak, 1972, 12, 297.

Immorlica, N. and M. Mahdian, "Marriage, Honesty, and Stability," in "Proceedings of the sixteenth annual ACM-SIAM symposium on Discrete algorithms" Society for Industrial and Applied Mathematics 2005, p. 62.

Jackson, M.O. and A.M. Manelli, "Approximately Competitive Equilibria in Large Finite Economies," Journal of Economic Theory, 1997, 77 (2), 354-376.

Kelso, A.S. and V.P. Crawford, "Job Matching, Coalition Formation, and Gross Substitutes," Econometrica, 1982, 50 (6), 1483-1504.

Kesten, O. and M.U. Ünver, "A Theory of School Choice Lotteries," 2010. Mimeo, Boston College.

Klemperer, P.D. and M.A. Meyer, "Supply Function Equilibria in Oligopoly under Uncertainty," Econometrica, 1989, pp. 1243-1277.

Knuth, D.E., Mariages Stables Et Leurs Relations Avec D'Autres Problèmes Combinatoires: Introduction À L'Analyse Mathématique Des Algorithmes, Presses de l'Université de Montréal, 1976.

Kojima, F. and M. Manea, "Incentives in the Probabilistic Serial Mechanism," Journal of Economic Theory, 2009.

and P.A. Pathak, "Incentives and Stability in Large Two-Sided Matching Markets," American Economic Review, 2009, 99 (3), 608-627.

, and A.E. Roth, "Matching with Couples: Stability and Incentives in Large Markets," 2010. NBER Working Paper No. 16028.

Lee, S., M. Niederle, H. Kim, and U. Kim, "Propose with a Rose? Signaling in Internet Dating Markets," 2011. NBER Working Paper No. 17340.

Lee, S.M., "Incentive Compatibility of Large Centralized Matching Markets," 2011. Mimeo, Caltech.

Manea, M., "Asymptotic Ordinal Inefficiency of Random Serial Dictatorship," Theoretical Economics, 2009, 4 (2), 165-197.

Mas-Colell, A., The Theory of General Economic Equilibrium: a Differentiable Approach, Cambridge Univ Press, 1990.

Milnor, J.W., Topology from the Differentiable Viewpoint, Princeton Univ Pr, 1997.

Mortensen, D.T. and C.A. Pissarides, "Job Creation and Job Destruction in the Theory of Unemployment," Review of Economic Studies, 1994, 61 (3), 397.

Niederle, M. and L. Yariv, "Decentralized Matching with Aligned Preferences," 2009. NBER Working Paper Number 14840.

Ostrovsky, M., "Stability in Supply Chain Networks," American Economic Review, 2008, 98 (3), 897-923. 
Oyer, P. and S. Schaefer, "Firm/Employee Matching: An Industry Study of American Lawyers," 2010. Mimeo, Stanford Graduate School of Business.

Pesendorfer, W. and J.M. Swinkels, "Efficiency and Information Aggregation in Auctions," American Economic Review, 2000, pp. 499-525.

Pollard, D., Convergence of Stochastic Processes, Springer, 1984.

Pycia, M. and Q. Liu, "Ordinal Efficiency, Fairness, and Incentives in Large Markets," 2011. Mimeo, UCLA.

Reny, P.J. and M. Perry, "Toward a Strategic Foundation for Rational Expectations Equilibrium," Econometrica, 2006, 74 (5), 1231-1269.

Roberts, D.J. and A. Postlewaite, "The Incentives for Price-Taking Behavior in Large Exchange Economies," Econometrica:, 1976, pp. 115-127.

Roth, A.E., "The Economics of Matching: Stability and Incentives," Mathematics of Operations Research, 1982, pp. 617-628.

, "Stability and Polarization of Interests in Job Matching," Econometrica, 1984, pp. $47-57$.

,"The College Admissions Problem is Not Equivalent to the Marriage Problem," Journal of Economic Theory, 1985, 36 (2), 277-288.

and E. Peranson, "The Redesign of the Matching Market for American Physicians: Some Engineering Aspects of Economic Design," American Economic Review, 1999, 89 (4), 748-780.

and M. Sotomayor, "The College Admissions Problem Revisited," Econometrica, 1989, 57 (3), 559-570.

Samet, D., "Matching of like Rank and the Size of the Core in the Marriage Problem," Mimeo, Tel Aviv University, 2010.

Sasaki, H. and M. Toda, "Two-Sided Matching Problems with Externalities," Journal of Economic Theory, 1996, 70 (1), 93-108.

Segal, I., "The communication requirements of social choice rules and supporting budget sets," Journal of Economic Theory, 2007, 136 (1), 341-378.

Sönmez, T., "Bidding for Army Career Specialties: Improving the Rotc Branching Mechanism," 2011. Mimeo, Boston College.

and T. Switzer, "Matching with (Branch-of-Choice) Contracts at United States Military Academy," 2011. Mimeo, Boston College.

Sorensen, M., "How Smart is Smart Money? A Two-Sided Matching Model of Venture Capital," Journal of Finance, December 2007, 62 (6), 2725.

Spence, A.M., "Monopoly, Quality, and Regulation," Bell Journal of Economics, 1975, pp. 417-429.

Starr, R.M., "Quasi-Equilibria in Markets with Non-Convex Preferences," Econometrica, 1969, pp. 25-38. 
Swinkels, J.M., "Efficiency of Large Private Value Auctions," Econometrica, 2001, 69 (1), 37-68.

Tervio, M., "The Difference that Ceos Make: An Assignment Model Approach," American Economic Review, 2008, 98 (3), 642-668.

Tiebout, C.M., "A Pure Theory of Local Expenditures," Journal of Political Economy, 1956, pp. 416-424.

Vapnik, V.N. and A.Y. Chervonenkis, "On the Uniform Convergence of Relative Frequencies of Events to Their Probabilities," Theory of Probability and its Applications, 1971, 16, 264.

Veiga, A. and E.G. Weyl, "Multidimensional Heterogeneity and Platform Design," 2011. http://papers.ssrn.com/sol3/papers.cfm?abstract_id=1935912. 


\section{Appendix}

This Appendix includes proofs of the results in the text, as well as additional results which are necessary to derive the results in the text. The Appendix is organized as follows. Appendix A extends some results of classic matching theory to the continuum model. It proves convergence of the analogue of the Gale and Shapley deferred acceptance algorithm, existence of a stable matching, the Lattice Theorem, and the Rural Hospitals Theorem. It also contains a proof of the continuum Supply and Demand Lemma. Appendix B derives our main results. Appendix C collects other proofs that have been omitted from the main text. Finally, Appendix D gives an example clarifying the difference between our characterization of stable matchings and Adachi's (2000).

\section{Appendix A. Preliminary Results}

We begin the analysis by deriving some basic properties of the set of stable matchings in the continuum model. Besides being of independent interest, they will be useful in the derivation of the main results. Throughout this section we fix a continuum economy $E=[\eta, S]$, and omit dependence on $E, \eta$, and $S$ in the notation.

A.1. Existence of a stable matching. We begin by proving the existence of a stable matching. Following the classic proof by Gale and Shapley (1962), we do so by defining the continuum analogue of the Gale and Shapley algorithm, and proving that it converges to a stable matching.

The continuum version of the Gale and Shapley student proposing algorithm is defined as follows. The state of the algorithm at round $k$ is a (not necessarily stable) matching $\mu^{k}$, a list $r^{k}(\theta)$ of colleges that have rejected each student $\theta$ so far, and a vector $x^{k}$ of cutoffs. The algorithm starts with the matching $\mu^{0}$ where all students unassigned, no rejections $r^{0}(\cdot) \equiv \emptyset$, and $x_{c}^{0} \equiv 0$. In each round, the state is updated as follows.

- Step 1: Each student that is unassigned at $\mu^{k}$ is tentatively assigned to her favorite college that hasn't rejected her yet, if there are any.

- Step 2: Each college rejects all students strictly below the minimum threshold score $x_{c}^{k+1} \geq x_{c}^{k}$ such that the measure of students assigned to it is smaller or equal to $S_{c} \cdot \mu^{k+1}$ is defined by this matching, and $r^{k+1}$ updated with the corresponding rejections.

We have that, although the algorithm does not necessarily finish in a finite number of steps, the tentative assignments converge to a stable matching.

Proposition A1. (Deferred Acceptance Convergence) The student-proposing deferred acceptance algorithm converges pointwise to a stable matching. 
Proof. To see that the algorithm converges, note that each student can only be rejected at most $C$ times. Consequently, for every student there exists $k$ high enough such that in all rounds of the algorithm past $k$ she is assigned the same college or matched to herself, so the pointwise limit exists. Therefore, there exists a function $\mu: \Theta \rightarrow C \cup\{\Theta\}$ that is the pointwise limit $\mu(\theta)=\lim _{k \rightarrow \infty} \mu^{k}(\theta)$. We may extend $\mu$ to $C$ by setting $\mu(c)=\{\theta \in \Theta: \mu(\theta)=c\}$. To see that the limit $\mu$ is a matching, we have to prove that the measure of students assigned to each college in the limit is not greater than its capacity. At each round $k$ of the algorithm, let $R^{k}$ be the measure of rejected students. Again, because no student can be rejected more than $C$ times, we have $R^{k} \rightarrow 0$. Moreover, it must be the case that $\eta(\mu(c)) \leq \eta\left(\mu^{k}(c)\right)+R_{k} \leq S_{c}+R^{k}$ for every $k$. Therefore, $\eta(\mu(c)) \leq S_{c}$.

Note that $\mu$ satisfies the consistency conditions 1-3 for a stable matching. Condition 4 (right continuity) follows from the fact that sets of rejected students are always of the form $e_{c}^{\theta}<x$. Therefore, $\mu$ is a matching.

The proof that the matching $\mu$ is stable follows Gale and Shapley (1962). Assume by contradiction that $(\theta, c)$ is a blocking pair. If $\eta(\mu(c))<S_{c}$, then $c$ does not reject any students during the algorithm, which contradicts $(\theta, c)$ being a blocking pair. This implies that there is $\theta^{\prime}$ in $\mu(c)$ with $e_{c}^{\theta^{\prime}}<e_{c}^{\theta}$. At some round $k$ of the algorithm, both agents are already matched to their final outcomes. But because $\theta$ was rejected by $c$ in an earlier round, it must be that $x_{c}^{k}>e_{c}^{\theta}$. Therefore $e_{c}^{\theta^{\prime}}$ would have to be rejected at round $k$, which is a contradiction.

The Proposition shows that the traditional algorithm for finding stable matchings works in the continuum model, although the algorithm converges without necessarily finishing in a finite number of steps. An immediate corollary of this Proposition is that stable matchings always exist. ${ }^{38}$

Corollary A1. (Existence) There exists at least one stable matching.

A.2. The Supply and Demand Lemma. We prove the continuum Supply and Demand Lemma 1.

Proof. (Lemma 1) Let $\mu$ be a stable matching, and $P=\mathcal{P} \mu$. Consider a student $\theta$ with $\mu(\theta)=c$. By definition, $P_{c} \leq e_{c}^{\theta}$. Consider a college $c^{\prime}$ that $\theta$ prefers over $c$. By right continuity, there is a student $\theta_{+}=\left(\succ^{\theta}, e^{\theta_{+}}\right)$with slightly higher scores than $\theta$ that is matched to $c$ and prefers $c^{\prime}$. By stability of $\mu$ all the students that are matched to $c^{\prime}$ have higher $c^{\prime}$ scores than $\theta_{+}$, so $P_{c^{\prime}} \geq e_{c^{\prime}}^{\theta_{+}}>e_{c^{\prime}}^{\theta}$. Following the argument for all colleges that $\theta$ prefers to $c$, we see that there are no colleges that are better than $c$

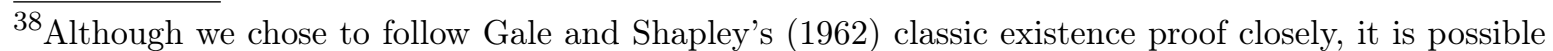
to give a shorter existence proof using Tarski's fixed point Theorem. We follow these lines in the extension to matching with contracts.
} 
and that $\theta$ can afford at cutoffs $P$. Therefore, $c$ is better than any other college that $\theta$ can afford, so $D^{\theta}(P)=\mu(\theta)$. This implies that no school is over-demanded given $P$, and that $\mathcal{M P} \mu=\mu$. To conclude that $P$ is a market clearing cutoff, note that if $\eta(\mu(c))<S_{c}$ stability implies that a student whose first choice is $c$ and has score at $c$ of zero is matched to $c$. Therefore, $P_{c}=0$.

To prove the other direction of the Lemma, let $P$ be a market clearing cutoff, and $\mu=\mathcal{M} P$. By the definition of $D^{\theta}(P), \mu$ is right-continuous and measurable. Because $P$ is a market clearing cutoff, $\mu$ respects capacity constraints. It respects the consistency conditions to be a matching by definition. To show that $\mu$ is stable, consider any potential blocking pair $(\theta, c)$ with $\mu(\theta) \prec^{\theta} c$. Since $\theta$ does not demand $c$ (i.e. $\mu(\theta)=$ $\left.D^{\theta}(P) \neq c\right)$, it must be that $P_{c}>e_{c}^{\theta}$, so $P_{c}>0$ and $c$ has no empty seats. If $\theta^{\prime} \in \mu(c)$ we have $e_{c}^{\theta^{\prime}} \geq P_{c}>e_{c}^{\theta}$, and therefore $(\theta, c)$ is not a blocking pair. Thus $\mu$ is stable. Let $P^{\prime}=\mathcal{P} \mu$. If $\mu(\theta)=c$, then $e_{c}^{\theta} \geq P_{c}$. Therefore, $P_{c}^{\prime} \geq P_{c}$. However, if $\theta$ is a student with $e_{c}^{\theta}=P_{c}$ whose favorite college is $c$, then $\mu(\theta)=D^{\theta}(P)=c$. Therefore $P_{c}^{\prime} \leq P_{c}$. These two inequalities imply that $P^{\prime}=P$, and therefore $\mathcal{P} \mathcal{M} P=P$.

A.3. Lattice Theorem and Rural Hospitals Theorem. Consider the sup $(\vee)$ and $\inf (\wedge)$ operators on $\mathbb{R}^{n}$ as lattice operators on cutoffs. That is, given two vectors of cutoffs

$$
\left(P \vee P^{\prime}\right)_{c}=\sup \left\{P_{c}, P_{c}^{\prime}\right\}
$$

More generally, given an arbitrary set of cutoffs $X \subseteq 2^{\left([0,1]^{C}\right)}$, we define the sup and inf operators analogously. That is

$$
(\vee X)_{c}=\sup _{P \in X} P_{c}
$$

We then have that the set of market clearing cutoffs forms a complete lattice with respect to these operators.

Theorem A1. (Lattice Theorem) The set of market clearing cutoffs is a complete lattice under $\vee, \wedge$.

Proof. First note that the set of market clearing cutoffs is nonempty. Now consider two market clearing cutoffs $P$ and $P^{\prime}$, and let $P^{+}=P \vee P^{\prime}$. Take a college $c$, and assume without loss of generality that $P_{c} \leq P_{c}^{\prime}$. By the definition of demand, we must have that $D_{c}\left(P^{+}\right) \geq D_{c}\left(P^{\prime}\right)$, as $P_{c}^{+}=P_{c}^{\prime}$ and the cutoffs of other colleges are higher under $P^{+}$. Also, if $P_{c}^{\prime}>0$, then $D_{c}\left(P^{+}\right) \geq q_{c} \geq D_{c}(P)$. Moreover, if $P_{c}^{\prime}=0$, then $P_{c}=P_{c}^{\prime}$, and $D_{c}\left(P^{+}\right) \geq D_{c}(P)$. Either way, we have that

$$
D_{c}\left(P^{+}\right) \geq \max \left\{D_{c}(P), D_{c}\left(P^{\prime}\right)\right\} .
$$

Moreover, the demand for staying unmatched $1-\sum_{c \in C} D_{c}(\cdot)$ must at least as large under $P^{+}$than under $P$ or $P^{\prime}$. Because demand for staying unmatched plus for all 
colleges always sums to 1 , we have that for all colleges $D_{c}\left(P^{+}\right)=D_{c}(P)=D_{c}\left(P^{\prime}\right)$. In particular $P^{+}$is a market clearing cutoff. The proof for the inf operator is analogous. This establishes that the set of market clearing cutoffs is a lattice.

This Theorem imposes a strict structure in the set of stable matchings. It differs from the Conway lattice Theorem in the discrete setting (Knuth 1976), as the set of stable matchings forms a lattice with respect to the operation of taking the sup of the associated cutoff vectors. In the discrete model, where the sup of two matchings is defined as the matching where each student gets her favorite college in each of the matchings. Such a Proposition does not carry over to the continuum model.

As a direct corollary of the proof we have the following.

Theorem A2. (Rural Hospitals Theorem) The measure of students matched to each college is the same in any stable matching. Furthermore, if a college does not fill its capacity, it is matched to the same set of students in every stable matching, except for a set of students with $\eta$ measure 0 .

Proof. The first part was proved in the proof of Theorem A1. To see the second part, consider two stable matchings $\mu$ and $\mu^{\prime}$. Let $P=\mathcal{P} \mu, P^{\prime}=\mathcal{P} \mu^{\prime}$. Let $P^{+}=P \vee P^{\prime}$ and $\mu^{+}=\mathcal{M}\left(P^{+}\right)$. Consider now a college $c$ such that $\eta(\mu(c))<S_{c}$. Therefore $0=P_{c}=P_{c}^{\prime}=\max \left\{P_{c}, P_{c}^{\prime}\right\}=P_{c}^{+}=0$. By the gross substitutes property of demand we have that $\mu(c) \subseteq \mu^{+}(c)$ and $\mu^{\prime}(c) \subseteq \mu^{+}(c)$. By the first part of the Theorem we know that the measure of $\mu(c), \mu^{\prime}(c)$, and $\mu^{+}(c)$ are the same. Therefore, $\eta\left(\mu^{+}(c) \backslash \mu(c)\right)=0$. Consequently, $\eta\left(\mu(c) \backslash \mu^{\prime}(c)\right) \leq \eta\left(\mu^{+}(c) \backslash \mu^{\prime}(c)\right)=0$. Using a symmetric argument we get that $\eta\left(\mu^{\prime}(c) \backslash \mu(c)\right)=0$, completing the proof.

This result implies that a hospital that does not fill its quota in one stable matching does not fill its quota in any other stable matching. Moreover, the measure of unmatched students is the same in every stable matching.

\section{Appendix B. Main Results}

B.1. Uniqueness. We can now prove Theorem 1.We denote the excess demand given a vector of cutoffs $P$ and an economy $E=[\eta, S]$ by

$$
z(P \mid E)=D(P \mid \eta)-S
$$

Proof. (Theorem 1)

Part (1):

By the lattice Theorem, $E$ has smallest and greatest market clearing cutoffs $P^{-} \leq$ $P^{+}$, and corresponding stable matchings $\mu^{-}, \mu^{+}$. In the text, we prove that there is a unique stable matching when $P^{+}>0$. We now consider the general case, where it may be that for some colleges $P_{c}^{+}=0$. Let $C^{+}=\left\{c \in C: P_{c}^{+}>0\right\}$ and $C^{0}=C \backslash C^{+}$. If $C^{+}$ 
is empty, then $P^{-}=P^{+}=0$, and we are done. Note that, since for all colleges $c \in C^{0}$ we have $P_{c}^{+}=P_{c}^{-}=0$, and for all colleges $c$ in $C^{+}$we have $P_{c}^{+} \geq P_{c}^{-}$, we have that

$$
\left\{\theta \in \Theta: \mu^{+}(\theta) \in C^{+}\right\} \subseteq\left\{\theta \in \Theta: \mu^{-}(\theta) \in C^{+}\right\} .
$$

By the Rural Hospitals Theorem, the difference between these two sets must have measure 0 . That is

$$
\eta\left(\left\{\theta \in \Theta: \mu^{-}(\theta) \in C^{+}\right\} \backslash\left\{\theta \in \Theta: \mu^{+}(\theta) \in C^{+}\right\}\right)=0 .
$$

Let $\succ^{+}$be a fixed preference relation that ranks all colleges in $C^{+}$higher than those in $C^{0}$. Then the set in the above equation must contain all students with preference $\succ^{+}$and scores $P_{c}^{-} \leq e_{c}^{\theta}<P_{c}^{+}$for all $c \in C^{+}$. That is,

$$
\begin{array}{r}
\left\{\left(\succ^{+}, e^{\theta}\right) \in \Theta: P_{c}^{-} \leq e_{c}^{\theta}<P_{c}^{+}\right\} \\
\subseteq\left\{\theta \in \Theta: \mu^{-}(\theta) \in C^{+}\right\} \backslash\left\{\theta \in \Theta: \mu^{+}(\theta) \in C^{+}\right\} .
\end{array}
$$

Therefore, the measure of this set must be 0 :

$$
\eta\left(\left\{\left(\succ^{+}, e^{\theta}\right) \in \Theta: P_{c}^{-} \leq e_{c}^{\theta}<P_{c}^{+}\right\}\right)=0 .
$$

By the full support assumption, this can only be the case if $P_{c}^{-}=P_{c}^{+}$for all $c \in C^{+}$. Since $P_{c}^{-}=P_{c}^{+}$for $c \in C^{0}$, we have that $P^{-}=P^{+}$, and therefore there exists a unique vector of market clearing cutoffs.

\section{Part (2):}

The proof is based on Sard's Theorem, from differential topology. Since we assume $\sum S_{i}<1$, we have that at all market clearing cutoffs $z(P)=0 .{ }^{39}$ Then, by Sard's Theorem, for generic $S$, every market clearing cutoff is a regular point of $z(\cdot \mid E){ }^{40}$ That is, the derivative of $z$ at every market clearing cutoff is invertible. This property will be crucial to proving the Theorem. To take into account the cases where some colleges have market clearing cutoffs of 0 , and may be in excess supply, we have to define the following auxiliary functions. We will reach a contradiction by showing that if $E$ has multiple market clearing cutoffs, then at least one of them has a singular derivative matrix.

Formally, consider a capacity vector $S$ such that market clearing cutoffs are regular points of $z$. By Sard's Theorem, this is the case for almost every $S$. To reach a

\footnotetext{
${ }^{39}$ See Guillemin and Pollack (1974); Milnor (1997). Consider a $C^{1}$ function $f: \mathbb{R}^{n} \rightarrow \mathbb{R}^{n}$. Sard's Theorem says that, for generic $q$, all the roots of $f(x)=q$ have an invertible derivative. That is, if $x_{0}$ is a root, then $\partial_{x} f\left(x_{0}\right)$ is nonsingular.

${ }^{40}$ Here is a detailed argument. We have $z(P \mid E)=D(P \mid \eta)-S$. Consequently, the roots of $z$ are the points where $D(P \mid \eta)=S$. Denote by $\bar{P}_{0}$ the closure of the set of points $P$ where $D$ is not continuously differentiable. By the regularity assumption, $D\left(\bar{P}_{0} \mid \eta\right)$ has measure 0 . Let $\bar{P}_{1}$ be the set of critical points of $D$ in $[0,1]^{n} \backslash \bar{P}_{0}$. By Sard's Theorem, its image $D\left(\bar{P}_{1} \mid \eta\right)$ has measure 0. Therefore, almost every $S$ is not in the image of either $\bar{P}_{0}$ nor $\bar{P}_{1}$, and so it is a regular value of $D(P \mid \eta)$. Because $z(P \mid E)=D(P \mid \eta)-S, 0$ is a regular value of $z$ for generic $S$.
} 
contradiction, assume that the economy $[\eta, S]$ has more than one stable matching. Let $P^{-} \neq P^{+}$be the minimum and maximum market clearing cutoffs. We will show $P^{-}$is not a regular point of $z$, reaching a contradiction.

First we consider the case $P_{c}^{-}<P_{c}^{+}$for all $c=1, \ldots, n$. Consider the cube $\{P \in$ $\left.[0,1]^{C} \mid P^{-} \leq P \leq P^{+}\right\}$. For any $P$ in the cube, we have $P^{-} \leq P \leq P^{+}$. Therefore $0=\sum_{c} z_{c}\left(P^{-}\right) \geq \sum_{c} z_{c}(P) \geq \sum_{c} z_{c}\left(P^{+}\right)=0$. This implies that the sum $\sum_{c} z_{c}(P)$ is constant on the cube. Therefore the derivative matrix of $P^{-}$satisfies $\partial_{P} z\left(P^{-}\right) \cdot \overrightarrow{1}=0$, and is singular. This implies that $P^{-}$is not a regular point of $z$, reaching a contradiction.

We now turn to the case where some colleges have the same cutoffs at $P^{-}$and $P^{+}$. Assume without loss of generality that $P_{c}^{-}<P_{c}^{+}$for colleges $c=1, \ldots, l$ and $P_{c}^{-}=P_{c}^{+}$for colleges $c=l+1, \ldots, C$. Let $P$ be a cutoff vector in the $l$-dimensional cube $X=\left\{P \in[0,1]^{C} \mid P^{-} \leq P \leq P^{+}\right\}$. For any school $c \in\{l+1, \cdots, C\}$, since $P_{c}^{+}=P_{c}^{-}=P_{c}$, we must have $z_{c}\left(P^{-}\right) \geq z_{c}(P) \geq z_{c}(P)$. However, by the Rural Hospitals Theorem, we have $z_{c}\left(P^{-}\right)=z_{c}\left(P^{+}\right)$, and therefore $z_{c}(P)=z_{c}\left(P^{-}\right)=z_{c}\left(P^{+}\right)$. This means that $z_{c}(\cdot)$ is constant on the cube. In particular, for any $c \in\{1,2, \cdots, l\}$ and $c^{\prime} \in\{l+1, l+2, \cdots, C\}$ and $P \in X$ in the cube must be the case that

$$
\partial_{c} z_{c^{\prime}}(P)=0 \text {. }
$$

This implies that the derivative matrix $\partial_{P} z(P)$ takes the subspace $F=\left\{v \in \mathbb{R}^{C}\right.$ : $\left.v_{l+1}=v_{l+2}=\cdots=v_{C}=0\right\}$ in itself. We now consider how $\partial_{P} z$ operates within $F$.

By the gross substitutes property, we have that. for all $P \in X$ in the cube,

$$
\sum_{c=1, \cdots, l} z_{c}\left(P^{-}\right) \geq \sum_{c=1, \cdots, l} z_{c}(P) \geq \sum_{c=1, \cdots, l} z_{c}\left(P^{+}\right) .
$$

However, for any college $c \in\{1, \cdots, l\}$, since $P_{c}^{+}>0$, by the market clearing condition and the Rural Hospitals Theorem we have that $z_{c}\left(P^{-}\right)=z_{c}\left(P^{+}\right)=0$. Therefore for any $P \in X$ we have

$$
\sum_{c=1, \cdots, l} z_{c}\left(P^{-}\right)=\sum_{c=1, \cdots, l} z_{c}(P)=\sum_{c=1, \cdots, l} z_{c}\left(P^{+}\right) .
$$

Therefore, the sum $\sum_{c=1, \cdots, l} z_{c}(P)$ is constant in the cube $X$. In particular, $\partial_{c^{\prime}} \sum_{c=1, \cdots, l}$ $z_{c}(P)=0$, for all $c^{\prime} \in\{1, \cdots, l\}$. Therefore, the matrix $\partial_{P} z(P)$ restricted to the subspace $F$ is not invertible. Since $\partial_{P} z(P)$ takes $F$ into itself, then $\partial_{P} z(P)$ is not invertible. In particular, $\partial_{P} z\left(P^{-}\right)$is not invertible, reaching a contradiction.

\section{B.2. Continuity and convergence.}

B.2.1. Continuity Within $\mathcal{E}$. This Section establishes that the stable matching correspondence is continuous around an economy $E \in \mathcal{E}$ with a unique stable matching. That is, that if a continuum economy has a unique stable matching, it varies continuously with the fundamentals. 
Note that, by our definition of convergence, we have that if the sequence of continuum economies $\left\{E^{k}\right\}_{k \in \mathbb{N}}$ converges to a continuum economy $E$, then the functions $z\left(\cdot \mid E^{k}\right)$ converge pointwise to $z(\cdot \mid E)$. Moreover, using the assumption that firms' indifference curves have measure 0 at $E$, we have the following Lemma.

Lemma B1. Consider a continuum economy $E=[\eta, S]$, a vector of cutoffs $P$ and a sequence of cutoffs $\left\{P^{k}\right\}_{k \in \mathbb{N}}$ converging to $P$. If $\left\{\eta^{k}\right\}_{k \in \mathbb{N}}$ converges to $\eta$ in the weak-* sense and $S^{k}$ converges to $S$ then

$$
z\left(P^{k} \mid\left[\eta^{k}, S^{k}\right]\right)=D\left(P^{k} \mid \eta^{k}\right)-S^{k}
$$

converges to $z(P \mid E)$.

Proof. Let $G^{k}$ be the set

$$
\cup_{c}\left\{\theta \in \Theta:\left|e_{c}^{\theta}-P_{c}\right| \leq \sup _{k^{\prime} \geq k}\left|P_{c}^{k^{\prime}}-P_{c}\right|\right\} .
$$

The set

$$
\cap_{k} G^{k}=\cup_{c}\left\{\theta \in \Theta: e_{c}^{\theta}=P_{c}\right\},
$$

has $\eta$-measure 0 by the strict preferences assumption 1 . Since the $G^{k}$ are nested, we have that $\eta\left(G^{k}\right)$ converges to 0 as $k \rightarrow \infty$.

Now take $\epsilon>0$. There exists $k_{0}$ such that for all $k \geq k_{0}$ we have $\eta\left(G^{k}\right)<\epsilon / 4$. Since the measures $\eta^{k}$ converge to $\eta$ in the weak sense, we may assume also that $\eta^{k}\left(G^{k_{0}}\right)<\epsilon / 2$. Since the $G^{k}$ are nested, this implies $\eta^{k}\left(G^{k}\right)<\epsilon / 2$ for all $k \geq k_{0}$. Note that $D^{\theta}(P)$ and $D^{\theta}\left(P^{k}\right)$ may only differ for $\theta \in G^{k}$. We have that

$$
\left|D(P \mid \eta)-D\left(P^{k} \mid \eta^{k}\right)\right|=\left|D(P \mid \eta)-D\left(P \mid \eta^{k}\right)\right|+\left|D\left(P \mid \eta^{k}\right)-D\left(P^{k} \mid \eta^{k}\right)\right| .
$$

As $\eta^{k}$ converges to $\eta$, we may take $k_{0}$ large enough so that the first term is less than $\epsilon / 2$. Moreover, since the measure $\eta\left(G^{k}\right)<\epsilon / 2$, we have that for all $k>k_{0}$ the second term is less than $\epsilon / 2$. Therefore, the above difference is less than $\epsilon$, completing the proof.

Note that this Lemma immediately implies the following:

Lemma B2. Consider a continuum economy $E=[\eta, S]$, a vector of cutoffs $P$ a sequence of cutoffs $\left\{P^{k}\right\}_{k \in \mathbb{N}}$ converging to $P$, and a sequence of continuum economies $\left\{E^{k}\right\}_{k \in \mathbb{N}}$ converging to $E$. We have that $z\left(P^{k} \mid E^{k}\right)$ converges to $z(P \mid E)$.

Using the Lemma, we show that the stable matching correspondence is upper hemicontinuous.

Proposition B1. (Upper Hemicontinuity) The stable matching correspondence is upper hemicontinuous 
Proof. Consider a sequence $\left\{E^{k}, P^{k}\right\}_{k \in \mathbb{N}}$ of continuum economies and associated market clearing cutoffs, with $E^{k} \rightarrow E$ and $P^{k} \rightarrow P$, for some continuum economy $E$ and vector of cutoffs $P$. We have $z(P \mid E)=\lim _{k \rightarrow \infty} z\left(P^{k}, E^{k}\right) \leq 0$. If $P_{c}>0$, for high enough $k$ we must have $P_{c}^{k}>0$ so that $z_{c}(P \mid E)=\lim _{k \rightarrow \infty} z_{c}\left(P^{k}, E^{k}\right)=0$.

With uniqueness, continuity also follows easily.

Lemma B3. (Continuity) Let $E$ be a continuum economy with a unique stable matching. Then the stable matching correspondence is continuous at $E$.

Proof. Let $P$ be the unique market clearing cutoff of $E$. Consider a sequence $\left\{E^{k}, P^{k}\right\}_{k \in \mathbb{N}}$ of economies and associated market clearing cutoffs, with $E^{k} \rightarrow E$. Assume, by contradiction that $P^{k}$ does not converge to $P$. Then $P^{k}$ has a convergent subsequence that converges to another point $P^{\prime} \in[0,1]^{C}$, with $P^{\prime} \neq P$. By the previous Proposition, $P^{\prime}$ must be a market clearing cutoff of $E$, contradicting the fact that $P$ is the unique market clearing cutoff of $E$.

B.2.2. Convergence of Finite Economics. We now consider the relationships between the stable matchings of a continuum economy, and stable matchings of a sequence of discrete economies that converge to it.

For finite economies $F$, we define the excess demand function as in the continuous case:

$$
z(P \mid F)=D(P \mid F)-S .
$$

Note that, with this definition, $P$ is a market clearing cutoff for finite economy $F$ iff $z(P \mid F) \leq 0$, with $z_{c}(P \mid F)=0$ for all colleges $c$ such that $P_{c}>0$.

From Lemma B1 we immediately obtain the following result.

Lemma B4. Consider a limit economy $E$, a sequence of cutoffs $\left\{P^{k}\right\}_{k \in \mathbb{N}}$ converging to $P$, and a sequence of finite economies $\left\{F^{k}\right\}_{k \in \mathbb{N}}$ converging to $E$. We then have that $z\left(P^{k} \mid F^{k}\right)$ converges to $z(P \mid E)$.

This Lemma then implies the following upper hemicontinuity property.

Proposition B2. (Convergence) Let $E$ be a continuum economy, and $\left\{F^{k}, P^{k}\right\}_{k \in \mathbb{N}}$ a sequence of discrete economies and associated market clearing cutoffs, with $F^{k} \rightarrow E$ and $P^{k} \rightarrow P$. Then $P$ is a market clearing cutoff of $E$.

Proof. (Proposition B2) We have $z(P \mid E)=\lim _{k \rightarrow \infty} z\left(P^{k} \mid F^{k}\right) \leq 0$. If $P_{c}>0$, then $P_{c}^{k}>0$ for large enough $k$, and we have $z_{c}(P \mid E)=\lim _{k \rightarrow \infty} z_{c}\left(P^{k} \mid F^{k}\right)=0$.

When the continuum economy has a unique stable matching, we can prove the stronger result below. 
Lemma B5. (Convergence with uniqueness) Let $E$ be a continuum economy with a unique market clearing cutoff $P$, and $\left\{F^{k}, P^{k}\right\}_{k \in \mathbb{N}}$ a sequence of discrete economies and associated market clearing cutoffs, with $F^{k} \rightarrow E$. Then $P^{k} \rightarrow P$.

Proof. (Lemma B5) To reach a contradiction, assume that $P^{k}$ does not converge to $P$. Then $P^{k}$ has a convergent subsequence that converges to another point $P^{\prime} \in[0,1]^{C}$. Then $z\left(P^{\prime} \mid E\right)=\lim _{k \rightarrow \infty} z\left(P^{k}, F^{k}\right) \leq 0$. If $P_{c}^{\prime}>0$, we must have that $P_{c}^{k}>0$ for all large enough $k$, and so $z_{c}\left(P^{\prime} \mid E\right)=0$. Therefore, $P^{\prime} \neq P$ is a market clearing cutoff, a contradiction.

B.2.3. Proof of Theorem 2. Theorem 2 follows from the previous results.

Proof. (Theorem 2) Part (3) follows from Lemma B3 and Part (1) follows from Lemma B2. As for Part (2), note first that given an economy $F^{k}$ the set of market clearing cutoffs is compact, which follows easily from the definition of market clearing cutoffs. Therefore, there exist market clearing cutoffs $P^{k}$ and $P^{\prime k}$ of $F^{k}$ such that the diameter of $F^{k}$ is $\left\|P^{k}-P^{\prime k}\right\|_{\infty}$. However, by Part (1), both sequences $\left\{P^{k}\right\}_{k \in \mathbb{N}}$ and $\left\{P^{\prime k}\right\}_{k \in \mathbb{N}}$ are converging to $P$, and therefore the diameter of $F^{k}$ is converging to 0 .

\section{Appendix C. Additional Proofs}

Proof. (Proposition 1)

First note that by our assumption that $f_{\delta}>0$ varies smoothly with $\delta$ and $\theta$ we have that $Y_{c}(P, e, \delta)$ is smooth. Aggregate quality is defined as

$$
\begin{aligned}
Q_{c}(\delta) & =\int_{\mu_{\delta}(c)} e_{c}^{\theta} d \eta_{\delta}(\theta) \\
& =\int_{\left\{\theta: D^{\theta}\left(P^{*}(\delta)\right)=c\right\}} e_{c}^{\theta} \cdot f_{\delta}(\theta) d \theta
\end{aligned}
$$

By Leibniz's rule, $Q_{c}$ is differentiable in $\delta_{c}$, and the derivative is given by

$$
\begin{aligned}
\frac{d Q_{c}(\delta)}{d \delta_{c}} & =\int_{\left\{\theta: D^{\theta}\left(P^{*}(\delta)\right)=c\right\}} e_{c}^{\theta} \cdot \frac{d}{d \delta_{c}} f_{\delta}(\theta) d \theta \\
& +\quad \sum_{c^{\prime} \neq c} \frac{d P_{c^{\prime}}^{*}}{d \delta_{c}} \cdot M_{c^{\prime} c} \cdot \bar{P}_{c^{\prime} c} \\
& -\frac{d P_{c}^{*}}{d \delta_{c}} \cdot\left[M_{c \emptyset}+\sum_{c^{\prime} \neq c} M_{c c^{\prime}}\right] \cdot P_{c}^{*} .
\end{aligned}
$$

The first term is the integral of the derivative of the integrand, and the last two terms the change in the integral due to the integration region $\left\{\theta: D^{\theta}\left(P^{*}(\delta)\right)=c\right\}$ changing with $\delta_{c}$. The terms in the second line are the changes due to changes in the cutoffs $P_{c^{\prime}}^{*}$, the students that school $c$ gains (or loses) because school $c^{\prime}$ becomes more (less) selective. The quantity of these students is $\frac{d P_{c^{\prime}}^{*}}{d \delta_{c}} \cdot M_{c^{\prime} c}$, and their average quality $\bar{P}_{c^{\prime} c}$. The last line is the term representing the students lost due to school $c$ raising its cutoff $P_{c}$. These students number $\left[M_{c \emptyset}+\sum_{c^{\prime} \neq c} M_{c^{\prime} c}\right]$, and have average quality $P_{c}^{*}$. Note that, 
since the total number of students admited at school $c$ is constant and equal to $S_{c}$, we have

$$
\begin{aligned}
0 & =\int_{\left\{\theta: D^{\theta}\left(P^{*}(\delta)\right)=c\right\}} \frac{d}{d \delta_{c}} f_{\delta}(\theta) d \theta \\
& +\quad \sum_{c^{\prime} \neq c} \frac{d P_{c^{\prime}}^{*}}{d \delta_{c}} \cdot M_{c^{\prime} c} \\
& -\quad \frac{d P_{c}^{*}}{d \delta_{c}} \cdot\left[M_{c \emptyset}+\sum_{c^{\prime} \neq c} M_{c c^{\prime}}\right] .
\end{aligned}
$$

Therefore, if we substitute $\frac{d P_{c}^{*}}{d \delta_{c}} \cdot\left[M_{c \emptyset}+\sum_{c^{\prime} \neq c} M_{c c^{\prime}}\right]$ in Equation (C.1) we have

$$
\begin{aligned}
\frac{d Q_{c}(\delta)}{d \delta_{c}} & =\int_{\left\{\theta: D^{\theta}\left(P^{*}(\delta)\right)=c\right\}}\left[e_{c}^{\theta}-P_{c}^{*}\right] \cdot \frac{d}{d \delta_{c}} f_{\delta}(\theta) d \theta \\
& +\quad \sum_{c^{\prime} \neq c} \frac{d P_{c^{\prime}}^{*}}{d \delta_{c}} \cdot M_{c^{\prime} c} \cdot\left[\bar{P}_{c^{\prime} c}-P_{c}^{*}\right] .
\end{aligned}
$$

The term in the second line is the market power effect as defined in the text. To see that the term in the first line is equal to the direct effect as defined in the text note that by definition of the function $Y(\cdot)$, we have

$$
\begin{aligned}
& \int_{\left\{\theta: D^{\theta}\left(P^{*}(\delta)\right)=c\right\}}\left[e_{c}^{\theta}-P_{c}^{*}\right] \cdot \frac{d}{d \delta_{c}} f_{\delta}(\theta) d \theta \\
= & \int_{e: e_{c} \geq P_{c}^{*}}\left[e_{c}-P_{c}^{*}\right] \cdot \frac{d Y_{c}}{d \delta_{c}}\left(P^{*}, \delta, e\right) d G(e) .
\end{aligned}
$$

To see that the direct effect is increasing, note that $u_{c}^{i}(\delta)$ is increasing in $\delta_{c}$ by assumption. Therefore, $\frac{d Y_{c}}{d \delta_{c}} \geq 0$.

\section{Additional details on Section 5.1.}

In Section 5.1 we gave a formula for the market power effect when the function $f_{\delta}(\theta)$ is symmetric over all schools, and schools choose the same level of quality. This formula follows from substituting an expression for $d P^{*} / d \delta_{c}$ in the formula for the market power term. To obtain the formula for $d P^{*} / d \delta_{c}$, we start from the point $\delta$ where all $\delta_{c}=\delta_{c^{\prime}}$. In this case, all $P_{c}^{*}(\delta)=P_{c^{\prime}}^{*}(\delta)$. If school $c$ changes $\delta_{c}$, the the cutoff $P_{c}^{*}(\delta)$ of school $c$ will change. The cutoffs of the other schools will change, but all other schools will have the same cutoff, which we term $P_{-c}^{*}(\delta)$. We denote $D_{c}\left(P_{c}, P_{-c} \mid \delta\right)$ for the demand for school $c$, and $D_{-c}\left(P_{c}, P_{-c} \mid \delta\right)$ for the demand for each other school under these cutoffs. Applying the implicit function theorem to the system of two equations

$$
\begin{aligned}
D_{c}\left(P_{c}, P_{-c} \mid \delta\right) & =S_{c} \\
D_{-c}\left(P_{c}, P_{-c} \mid \delta\right) & =S_{c} .
\end{aligned}
$$

we get

$$
\begin{aligned}
\frac{d}{d P_{c}} D_{c} \cdot \frac{d P_{c}}{d \delta_{c}}+\frac{d}{d P_{-c}} D_{c} \cdot \frac{d P_{c^{\prime}}}{d \delta_{c}}+\frac{d}{d \delta_{c}} D_{c}=0 \\
\frac{d}{d P_{c}} D_{c^{\prime}} \cdot \frac{d P_{c}}{d \delta_{c}}+\frac{d}{d P_{-c}} D_{c^{\prime}} \cdot \frac{d P_{c^{\prime}}}{d \delta_{c}}+\frac{d}{d \delta_{c}} D_{c^{\prime}}=0
\end{aligned}
$$


Substituting the derivative of the demand function as a function of the mass of agents on the margins $\tilde{M}_{c c^{\prime}}$, the system becomes

$$
\begin{aligned}
-\left[M_{c \emptyset}+(C-1) M_{c c^{\prime}}\right] \cdot \frac{d P_{c}}{d \delta_{c}}+\left[(C-1) M_{c^{\prime} c}\right] \cdot \frac{d P_{c^{\prime}}}{d \delta_{c}}+\frac{d}{d \delta_{c}} D_{c}=0 \\
{\left[M_{c c^{\prime}}\right] \cdot \frac{d P_{c}}{d \delta_{c}}-\left[M_{c^{\prime} \emptyset}+M_{c^{\prime} c}\right] \cdot \frac{d P_{c^{\prime}}}{d \delta_{c}}+\frac{d}{d \delta_{c}} D_{c^{\prime}}=0 . }
\end{aligned}
$$

Due to the symmetry of the problem, $M_{c c^{\prime}}=M_{c^{\prime} c}, M_{c \emptyset}=M_{c^{\prime} \emptyset}$, and $\frac{d}{d \delta_{c}} D_{c}=(C-$ 1) $\frac{d}{d \delta_{c}} D_{c^{\prime}}$. The formula in the text then follows from solving the system.

Proof. (Proposition 2) Note that since $\sum_{c} S_{c}<1$, market clearing cutoffs satisfy $z(P \mid E)=0$ In what follows we always take $\alpha$ to be large enough such that for any finite economy $F$ such that the bound in the Proposition has any content (that is, the right side is less than one), $\sum_{c} S_{c}^{F}<1$. This guarantees that market clearing cutoffs in such an economy must satisfy $z(P \mid F)=0$.

The proof begins by showing that at economy $E$, cutoffs $P$ that are far from the market clearing cutoff $P^{*}$ have large excess demands, in the sense that their norm is bounded below by a multiple of the distance to the market clearing cutoff $P^{*}$. Formally, let $B^{\epsilon}=\left\{P \in[0,1]^{C}:\left|P-P^{*}\right|<\epsilon\right\}$. Let

$$
\begin{aligned}
P^{\epsilon} & =\arg \min _{P \notin B^{\epsilon}}|z(P \mid E)| \text { and } \\
M^{\epsilon} & =\min _{P \notin B^{\epsilon}}|z(P \mid E)| .
\end{aligned}
$$

Note that, due to the continuity of the demand function, both $P^{\epsilon}$ and $M^{\epsilon}$ are well defined. Moreover, $P^{\epsilon}$ may be a set of values, in the case of multiple minima. In what follows, we will take a single-valued selection from this set, so that $P^{\epsilon}$ represents one of the minima. With this convention $M^{\epsilon}=\left|z\left(P^{\epsilon} \mid E\right)\right|$. We will now show that there exists $\alpha>0$ such that for all $0<\epsilon \leq 1$

$$
M^{\epsilon} \geq \frac{1}{\alpha} \cdot \epsilon
$$

To see this, note that since $D(\cdot \mid E)$ is $C^{1}$, we have that

$$
z(P \mid E)=D(P \mid E)-S=\partial D\left(P^{*} \mid E\right) \cdot\left(P-P^{*}\right)+o\left(P-P^{*}\right),
$$

where the continuous function $o(\cdot)$ satisfies that for any $\epsilon>0$ there exists $\delta>0$ such that for all $P \in B^{\delta}$

$$
\frac{\left|o\left(P-P^{*}\right)\right|}{\left|P-P^{*}\right|}<\epsilon .
$$

Since $\partial_{P} D\left(P^{*} \mid E\right)$ is nonsingular, we may take $A>0$ such that

$$
\left|\partial_{P} D\left(P^{*} \mid E\right) \cdot v\right| \geq 2 A \cdot|v|
$$

for any vector $v \in \mathbb{R}^{C}$. 
By the continuity condition, we may take $0<\epsilon_{0} \leq 1$ such that

$$
\frac{o\left(P-P^{*}\right)}{\left|P-P^{*}\right|}<A
$$

for all $P \in B^{\epsilon_{0}}$. Therefore, for all $P \in B^{\epsilon_{0}}$ we have

$$
\begin{aligned}
|z(P \mid E)| & =\left|\partial D\left(P^{*} \mid E\right) \cdot\left(P-P^{*}\right)+o\left(P-P^{*}\right)\right| \\
& \geq\left|\partial D\left(P^{*} \mid E\right) \cdot\left(P-P^{*}\right)\right|-\left|o\left(P-P^{*}\right)\right| \\
& \geq 2 A \cdot\left|P-P^{*}\right|-\left|\frac{o\left(P-P^{*}\right)}{\left|P-P^{*}\right|}\right| \cdot\left|P-P^{*}\right| \\
& \geq 2 A \cdot\left|P-P^{*}\right|-A \cdot\left|P-P^{*}\right| \\
& =A \cdot\left|P-P^{*}\right| .
\end{aligned}
$$

The first equality follows from the derivative formula for excess demand in Equation (C.2). The inequality in the second line follows from the triangle inequality. The inequality in the third line follows from the bound in Inequality (C.3) for the left term, and algebra for the right term. The inequality in the fourth line is a consequence of applying the continuity bound in Inequality (C.4) to the right term. Finally, the last line is straightforward algebra. The above reasoning establishes that for all $P \in B^{\epsilon_{0}}$ excess demand is bounded from below by

$$
|z(P \mid E)| \geq A \cdot\left|P-P^{*}\right|,
$$

which is linear on the norm $\left|P-P^{*}\right|$. In particular this implies that for all $0<\epsilon<\epsilon_{0}$ we have

$$
M^{\epsilon} \geq A \cdot \epsilon .
$$

We will now use this bound to obtain a bound that is valid for all $0<\epsilon \leq 1$. Take $\alpha>0$ such that

$$
\frac{1}{\alpha}=\min \left\{A, M^{\epsilon_{0}}\right\} .
$$

Therefore, if $0<\epsilon<\epsilon_{0}$ we have $M^{\epsilon} \geq A \cdot \epsilon \geq \frac{1}{\alpha} \cdot \epsilon$. If $\epsilon_{0} \leq \epsilon \leq 1$, then $M^{\epsilon} \geq M^{\epsilon_{0}} \geq$ $\frac{1}{\alpha} \geq \frac{1}{\alpha} \epsilon$. Either way, we have the desired bound

$$
M^{\epsilon} \geq \frac{1}{\alpha} \cdot \epsilon
$$

for all $0<\epsilon \leq 1$.

We now prove the Proposition. If $P^{F}$ is a market clearing vector of the finite economy $F$ then

$$
\left|z\left(P^{F} \mid E\right)-z\left(P^{F} \mid F\right)\right|=\left|z\left(P^{F} \mid E\right)\right| \geq \frac{1}{\alpha} \cdot\left|P^{F}-P^{*}\right| .
$$

The first equality follows from the definition of a market clearing cutoff, and the second by the bound for $M^{\epsilon}$ in Inequality (C.6). Moreover, by the triangle inequality we have 
that

$$
\left|z\left(P^{F} \mid E\right)-z\left(P^{F} \mid F\right)\right| \leq \mid \frac{D\left(P^{F} \mid \eta\right)-D\left(P^{F} \mid \eta^{F}\right)|+| S-S^{F} \mid}{\sup _{P \in[0,1]^{C}}\left|D(P \mid \eta)-D\left(P \mid \eta^{F}\right)\right|+\left|S-S^{F}\right| .} .
$$

Combining these two inequalities we obtain the desired bound

$$
\left|P^{F}-P^{*}\right| \leq \alpha \cdot\left(\sup _{P \in[0,1]^{C}}\left|D(P \mid \eta)-D\left(P \mid \eta^{F}\right)\right|+\left|S-S^{F}\right|\right) .
$$

Proof. (Proposition 3)

\section{Part (1): Almost sure convergence.}

First we show that that the sequence of random economies $\left\{F^{k}\right\}_{k \in \mathbb{N}}$ converges to $E$ almost surely. It is true by assumption that $S^{k}$ converges to $S$. Moreover, by the Glivenko-Cantelli Theorem, the realized measure $\eta^{K}$ converges to $\eta$ in the weak* topology almost surely. Therefore, by definition of convergence, we have that $F^{k}$ converges to $E$ almost surely. This implies, by Theorem 2, that $\mu^{k}$ converges to $\mu$.

Part (2): Bound on $\left|P^{*}-P^{k}\right|$.

We begin the proof by taking $\alpha_{0}$ as in Proposition 2 .

Let the agents in finite economy $F^{k}$ be $\theta^{1, k}, \theta^{2, k}, \cdots, \theta^{k, k}$. The demand function at economy $F^{k}$ is the random variable

$$
D_{c}\left(P \mid \eta^{k}\right)=\sum_{i=1, \cdots, k} 1_{\theta^{i, k} \in\left\{\theta \in \Theta: D^{\theta}(P)=c\right\}} / k .
$$

That is, $D_{c}$ are very similar to empirical distribution functions, measuring the fraction of agents $\theta^{i, k}$ whose types are in the set $\left\{\theta \in \Theta: D^{\theta}(P)=c\right\}$. By the Vapnik-Chervonenkis Theorem, ${ }^{41}$ there exists exists $\alpha$ such that the probability that

$$
\operatorname{Pr}\left\{\left|D\left(P \mid \eta^{k}\right)-D(P \mid \eta)\right|>\epsilon / 2 \alpha_{0}\right\} \leq \alpha_{1} \cdot \exp \left(-\frac{k}{8}\left(\frac{\epsilon}{2 \alpha_{0}}\right)^{2}\right) .
$$

Crucially, the Vapnik-Chervonenkis Theorem guarantees that this bound is uniform in $P$.

\footnotetext{
${ }^{41}$ See Theorem 12.5 in Devroye et al. (1996) pp. 197. As remarked in pp. 198, the bound given in pp. 197 is looser than the bound originally established by Vapnik and Chervonenkis (1971), which we use. The simple proof given in Devroye et al. (1996) follows the lines of Pollard (1984). The Theorem can be proven using Hoeffding's Inequality, and generalizes the Dvoretzky et al. (1956) inequality to the multidimensional case, and to arbitrary classes of measurable sets, not only sets of the form $\left\{x \in \mathbb{R}^{n}: x \leq \bar{x}\right\}$. The important requirement for the Theorem to apply in our setting is that the Vapnik-Chervonenkis dimension of the class of sets $\left\{\theta \in \Theta: D^{\theta}(P)=c\right\}$ is finite.
} 
If this is the case, by Proposition 2, the distance of all market clearing cutoffs $P^{k}$ of $F^{k}$ is bounded by

$$
\begin{aligned}
\left|P^{k}-P^{*}\right| & \leq \alpha_{0} \epsilon / 2 \alpha_{0}+\left|S-S^{k}\right| \\
& \leq \epsilon / 2+1 / k
\end{aligned}
$$

Therefore, for $k \geq k_{0}=\lceil 2 / \epsilon\rceil$,

$$
\left|P^{k}-P^{*}\right| \leq \epsilon
$$

Therefore, if we take

$$
\beta=\frac{1}{32 \alpha_{0}^{2}}
$$

and $\alpha \geq \alpha_{1}$ such that

$$
\alpha \beta^{k_{0}} \geq 1
$$

we have that the probability that $F^{k}$ has any market clearing cutoffs with $\left|P^{k}-P^{*}\right| \geq \epsilon$ is lower than $\alpha \beta^{k}$, as desired.

Part (3): Bound on $G^{k}$.

Let the set of agents with scores which have at least one coordinate close to $P_{c}^{*}$ be

$$
\bar{\Theta}=\left\{\theta \in \Theta: \exists c \in C:\left|e_{c}^{\theta}-P_{c}^{*}\right| \leq \epsilon / 4 C \bar{f}\right\}
$$

Let $\bar{f}$ be the supremum of the density of $\eta$. The $\eta$ measure of the set $\bar{\Theta}$ is bounded by

$$
\eta(\bar{\Theta}) \leq 2 C \bar{f} \cdot(\epsilon / 4 C \bar{f})=\epsilon / 2 .
$$

Let the agents in finite economy $F^{k}$ be $\theta^{1, k}, \theta^{2, k}, \cdots, \theta^{k, k}$. The fraction of agents in economy $F^{k}$ that have types in $\bar{\Theta}$ is given by the random variable

$$
G^{k}=\sum_{i=1, \cdots, k} 1_{\theta^{i, k} \in \bar{\Theta}} / k .
$$

By the Vapnik-Chervonenkis Theorem, in the argument of Part (2), we could have taken the constants $\alpha, \beta$ in a way that the probability that both the fraction of agents with types in $\bar{\Theta}$ differs from the expected number $\eta(\bar{\Theta}) \leq \epsilon / 2$ by more than $\epsilon / 2$ is lower than $\alpha \beta^{k} / 2$, and the probability

$$
\operatorname{Pr}\left\{\left|D\left(P \mid \eta^{k}\right)-D(P \mid \eta)\right|>\epsilon / 2 \alpha_{0}\right\} \leq \alpha \beta^{k} / 2 .
$$

If both events happen, then $G^{k}<\epsilon / 2+\epsilon / 2=\epsilon$. The probability that both events happen is at most $\alpha \beta^{k} / 2+\alpha \beta^{k} / 2=\alpha \beta^{k}$.

Proof. (Proposition 4) Consider the case where $P_{c}>P_{c}^{\prime}$, the other case is analogous. Let $\epsilon=\left|P_{c}-P_{c}^{\prime}\right|$. By Theorem 2 Part (3) the diameters of the set of stable matchings of economies in the sequences $F^{k}$ and $F^{\prime k}$ converge to 0 . Therefore, we may take $k_{0}$ 
large enough such that for any market clearing cutoffs $P^{k}, P^{\prime k}$ of $F^{k}, F^{\prime k}$ we have

$$
\begin{aligned}
& \left|P^{k}-P\right|<\epsilon / 2 \\
& \left|P^{\prime k}-P\right|<\epsilon / 2 .
\end{aligned}
$$

By the triangle inequality we then have

$$
P_{c}^{k}>P_{c}-\epsilon / 2 \geq P_{c}^{\prime}+\epsilon / 2>P_{c}^{\prime k}
$$

Proof. (Lemma 3) Let $\mu$ be a stable matching, and $P=\mathcal{P} \mu$. Consider a doctor $\theta$. Let $h_{0} \in H \cup\{\emptyset\}$ be the hospital to which she is matched to, or the empty set if she is unmatched. By definition of $\mathcal{P}$ we have that $u^{\theta}(\mu(\theta)) \leq \bar{u}_{h_{0}}^{\theta}(P)$. Let $x$ be any contract she strictly prefers to $\mu(\theta)$, in any hospital $h$ different than the one to which she is matched. By definition of stability, that hospital $h$ must be filling its quota, and for all contracts $x^{\prime} \in \mu(h)$ we must have $\pi_{h}(x) \leq \pi_{h}\left(x^{\prime}\right)$. Therefore, $\pi_{h}(x) \leq P_{h}$. Because this is true for any such contract $x, \bar{u}_{h}^{\theta}(P) \leq u^{\theta}(\mu(\theta)) \leq \bar{u}_{h_{0}}^{\theta}(P)$. By the strict preferences assumption, except for a measure 0 set we have $\bar{u}_{h}^{\theta}(P)<\bar{u}_{h_{0}}^{\theta}(P)$ for all agents $\theta$. Hence, for almost every agent,

$$
D^{\theta}(P)=\mu(\theta)
$$

and so aggregate demand satisfies $D(P) \leq S$. By the completeness assumption and stability, we must have that if $D_{h}(P)<S_{h}$, then for all $k>0$ hospital $h$ must be matched to the doctors in the definition of completeness. Therefore, $P_{h}=0$.

Now consider a market clearing cutoff $P$, and let $\mu=\mathcal{M} P . \mu$ is measurable by the measurability assumption. It is immediate that $\mu$ respects capacity constraints. It is also individually rational. Hence, we only have to show it has no blocking pairs. Assume, by contradiction, that $(\theta, h)$ is a blocking pair. Note that $h$ is only matched to contracts that give utility of at least $P_{h}$, by definition of $\mu$. If $\theta$ is matched to a hospital $h^{\prime}$ with a contract $x^{\prime}=\mu(\theta)$, then there exists a contract $x \in X_{h}^{\theta}$ such that

$$
\begin{aligned}
& u^{\theta}(x)>u^{\theta}\left(x^{\prime}\right) \\
& \pi_{h}(x) \geq P_{h} .
\end{aligned}
$$

In particular,

$$
\bar{u}_{h}^{\theta}(P)>u^{\theta}\left(x^{\prime}\right)
$$

However, this contradicts the definition of $\mathcal{M}$ in Equation (5.2). The case where $\theta$ is unmatched is analogous.

Proof. (Proposition 9) We begin by showing that the set of market clearing cutoffs is a lattice. To that end, define the operator $T:[0, M]^{H} \rightarrow[0, M]^{H}$ defined by $P^{\prime}=T P$ 
with $P_{h}$ being the solution to

$$
D_{h}\left(P_{h}^{\prime}, P_{-h}\right)=S_{h}
$$

if such a solution exists in $[0, M]$, and 0 otherwise. Note that, if the solution to this equation exists, it is unique, as the full support assumption implies that the left side is strictly decreasing in $P_{h}^{\prime}$. Also, because $D_{h}\left(M, P_{-h}\right)=0$, if the solution does not exist then $D\left(P_{h}^{\prime}, P_{-h}\right)<S_{h}$ for all $P_{h}^{\prime} \in[0, M]$. Therefore, the set of market clearing cutoffs is identical to the set of fixed points of $T$. Note that $T$ is weakly increasing in $P$, and takes $[0, M]$ in itself. Therefore, by Tarski's Theorem, the set of fixed points is a non-empty lattice.

Let $P^{-}$and $P^{+}$be the smallest and largest market clearing cutoffs. Let $H^{+}$be the subset of hospitals for which $P_{h}^{+}>0$, that is

$$
H^{+}=\left\{h \in H: P_{h}>0\right\} .
$$

If $H^{+}$is empty, then $P^{-}=P^{+}=0$, and we are done. Assume henceforth that $H^{+}$is nonempty. Then by the definition of a market clearing cutoff it must be the case that

$$
\sum_{h \in H^{+}} D_{h}\left(P^{-}\right) \leq \sum_{h \in H^{+}} S_{h}=\sum_{h \in H^{+}} D_{h}\left(P^{+}\right) .
$$

However, since $P_{h}^{-}=P_{h}^{+}$for $h \notin H^{+}$, and $P_{h}^{-}<P_{h}^{+}$for all $h \in H^{+}$we have that

$$
\sum_{h \in H^{+}} D_{h}\left(P^{-}\right) \geq \sum_{h \in H^{+}} D_{h}\left(P^{+}\right) .
$$

Therefore,

$$
\sum_{h \in H^{+}} D_{h}\left(P^{-}\right)=\sum_{h \in H^{+}} D_{h}\left(P^{+}\right) .
$$

Under the assumption that the support of $\eta_{s}$ is the set $[0, M]^{H}$, this can only be true if $P^{-}=P^{+}$, completing the proof.

\section{Appendix D. Pre-Matchings}

This Appendix clarifies the difference between our characterization of stable matchings and that of Adachi (2000); Echenique and Oviedo (2004) using pre-matchings. We give a simple example where the Adachi map takes a pre-matching that has a cutoff structure, and produces a pre-matching that does not have a cutoff structure. This clarifies how the Adachi operator approach requires keeping track of much more detailed information than simply cutoffs at each college.

Return to the discrete Gale and Shapley model of Section. Following Echenique and Oviedo (2004), we define a pre-matching as a map

$$
v: \tilde{\Theta} \cup C \rightarrow 2^{\tilde{\Theta}} \cup C
$$

such that 
(1) Each college is matched to a subset of students: $\forall c \in C: v(c) \in 2^{\tilde{\Theta}}$.

(2) Each student is matched to a college or to itself: $\forall \theta \in \tilde{\Theta}: v(\theta) \in C \cup\{\theta\}$.

Note that in a pre-matching a college may be matched to a set of students with more students than its capacity. Let $V$ be the set of all stable matchings. The Adachi operator $T: V \rightarrow V$ is defined as follows. Given a pre-matching $v, v^{\prime}=T v$ is given by

(1) For each college $c, v^{\prime}(c)$ is its favorite entering class out of the students who are matched to a worse college under $v$ :

$$
\left\{\theta: c \succ^{\theta} v(c)\right\}
$$

That is, $v^{\prime}(c)$ is the set of (at most) $S_{c} \cdot \# \tilde{\Theta}$ highest ranked students in this set.

(2) For each student, $v^{\prime}(c)$ is her favorite college out of those who would prefer to match with it given $v$. That is, he favorite college in the set

$$
\left\{c: \# v(c)<S_{c} \cdot \# \tilde{\Theta} \text {, or } c \text { prefers } \theta \text { to the } S_{c} \cdot \# \tilde{\Theta} \text { th ranked student in } v(c)\right\} \text {. }
$$

Consider now the following example. There are two colleges $C=\{1,2\}, S^{1}=S^{2}=1 / 3$ (meaning they have one position each, by our convention of using supply per student) and three students $\tilde{\Theta}=\left\{\theta^{1}, \theta^{2}, \theta^{3}\right\}$. Preferences are given by

$$
\begin{aligned}
& \succ^{\theta^{1}}=\succ^{\theta^{2}}=1,2 \\
& \succ^{\theta^{3}}=2,1 \\
& e^{\theta^{1}}=(1,1) \\
& e^{\theta^{2}}=(1 / 2,1 / 2) \\
& e^{\theta^{3}}=(0,0) .
\end{aligned}
$$

Consider the cutoffs $P=(0,0) . v=\mathcal{M} P$ is a pre-matching, given by

$$
\begin{aligned}
v\left(\theta^{1}\right) & =v\left(\theta^{2}\right)=1 \\
v\left(\theta^{3}\right) & =2 \\
v(1) & =\left\{\theta^{1}, \theta^{2}\right\}, v(2)=\left\{\theta^{3}\right\} .
\end{aligned}
$$

Although $v$ has more students assigned to college 1 than its capacity, it at least satisfies the consistency requirement (3) in the definition of a stable matching in the discrete model. Moreover, it has a cutoff structure, in the sense the $v=\mathcal{M} P$. We will now see that both these properties are lost if we apply the Adachi operator to $v$.

Let $v^{\prime}=T v$. We have

$$
\begin{array}{r}
v^{\prime}\left(\theta^{1}\right)=1, v^{\prime}\left(\theta^{2}\right)=2, v^{\prime}\left(\theta^{3}\right)=\theta^{3} \\
v^{\prime}(1)=\left\{\theta^{1}\right\}, v^{\prime}(2)=\left\{\theta^{3}\right\} .
\end{array}
$$


Note that this new pre-matching $v^{\prime}$ no longer satisfies the consistency requirement (3), as student 2 is pre-matched to college 2 , but college 2 is not pre-matched to student 2. Moreover, even if one tries to look at a consistent match as described by the pre matchings for one side, $\left(v^{\prime}(c)\right)_{c \in C}$, it does not have a cutoff structure. Given $\left(v^{\prime}(c)\right)_{c \in C}$, $\theta^{3}$ is matched to college 2. If there were cutoffs that described this allocation, this would mean $P_{2}=0$. However, $\theta^{2}$ is unmatched, which implies $P_{2}>1 / 2$.

The simple takeaway from this example is that the Adachi map $T$ does not preserve the cutoff structure. Therefore, in applying Adachi's algorithm one cannot simply keep track of a small number of cutoffs $P_{c}$ one for each college. This highlights the distinction between our and Adachi's approach, above the fact that we consider clearing supply and demand and Adachi considers fixed points of $T$. 